\title{
Well Posedness and Control in a NonLocal SIR Model
}

\author{
Rinaldo M. Colombo ${ }^{1} \quad$ Mauro Garavello ${ }^{2}$
}

October 17, 2019

\begin{abstract}
$S I R$ models, also with age structure, can be used to describe the evolution of an infective disease. A vaccination campaign influences this dynamics immunizing part of the susceptible individuals, essentially turning them into recovered individuals. We assume that vaccinations are dosed at prescribed times or ages which introduce discontinuities in the evolutions of the $S$ and $R$ populations. It is then natural to seek the "best" vaccination strategies in terms of costs and/or effectiveness. This paper provides the basic well posedness and stability results on the $S I R$ model with vaccination campaigns, thus ensuring the existence of optimal dosing strategies.
\end{abstract}

Keywords: Vaccination; Optimal Control of Balance Laws; Control in Age-Structured Populations Models.

2010 MSC: 35L65; 49J20; 92D30.

\section{Introduction}

Aim of this paper is to provide a rigorous analytic environment where different vaccination strategies can be described, tested and optimized.

Our starting point is the following age-structured Susceptible - Infected-Recovered $(S I R)$ model, which originated in [10, see also [9, Chapter 6], [14, Chapter 19] or [15, § 1.5.1],

$$
\begin{aligned}
& \partial_{t} S+\partial_{a} S=-d_{S}(t, a) S-\int_{0}^{+\infty} \lambda\left(a, a^{\prime}\right) I\left(t, a^{\prime}\right) \mathrm{d} a^{\prime} S \\
& \partial_{t} I+\partial_{a} I=-d_{I}(t, a) I+\underbrace{\int_{0}^{+\infty} \lambda\left(a, a^{\prime}\right) I\left(t, a^{\prime}\right) \mathrm{d} a^{\prime} S}_{\text {mortality }}-\underbrace{r_{I}(t, a) I}_{\text {disease transmission }}+\underbrace{r_{I}(t, a) I}_{\text {recovery }} . \\
& \partial_{t} R+\partial_{a} R=-\underbrace{d_{R}(t, a) R}_{R} .
\end{aligned}
$$

As usual, $S=S(t, a)$ is the density of individuals at time $t$ of age $a$ susceptible to the disease; $I=$ $I(t, a)$ is the density of infected individuals at time $t$ and of age $a$ and the density of individuals that can not be infected by the disease is $R=R(t, a)$, comprising individuals that recovered from the disease as well as those that are immune. The death rates of the three portions of the populations are $d_{S}, d_{I}$ and $d_{R}$. Above, $\lambda\left(a, a^{\prime}\right)$ quantifies the susceptible individuals of age $a$ that are infected by individuals of age $a^{\prime}$. Thus, the nonlocal term $\int_{0}^{+\infty} \lambda\left(a, a^{\prime}\right) I\left(t, a^{\prime}\right) \mathrm{d} a^{\prime} S(t, a)$ in the former two right hand sides represents the total number of susceptible individuals of age $a$

\footnotetext{
${ }^{1}$ INdAM Unit, University of Brescia. rinaldo.colombo@unibs.it

${ }^{2}$ Department of Mathematics and its Applications, University of Milano - Bicocca.

mauro.garavello@unimib.it
} 
that become infected at time $t$. Finally, $r_{I}(t, a)$ is the fraction of infected individuals of age $a$ that recover at time $t$, independently from the vaccination campaign.

We now introduce a vaccination campaign in (1.1). To this aim, differently from various paper in the literature, e.g. [7, [8, 12, 13, 18, 19, we do not introduce any source term in the right hand sides of (1.1). We consider two different approaches.

In a first policy, vaccinations are dosed at a, possibly time dependent, percentage of the population of the prescribed ages $\bar{a}_{1}, \bar{a}_{2}, \ldots, \bar{a}_{N}$, with $\bar{a}_{j-1}<\bar{a}_{j}$. Call $\eta_{j}(t)$, with $\eta_{j}(t) \in[0,1]$ the fraction of the $S$ population of age $\bar{a}_{j}$ that is dosed a vaccine at time $t$. Then, assuming that vaccination has an immediate effect, the evolution described by (1.1) has to be supplemented by the vaccination conditions

$$
\begin{array}{rlrl}
S\left(t, \bar{a}_{j}+\right) & =\left(1-\eta_{j}(t)\right) S\left(t, \bar{a}_{j}-\right) & {\left[\forall t, S\left(t, \bar{a}_{j}\right)\right. \text { decreases due to vaccination] }} \\
I\left(t, \bar{a}_{j}+\right) & =I\left(t, \bar{a}_{j}-\right) & \text { [the infected population is unaltered] } \\
R\left(t, \bar{a}_{j}+\right) & =R\left(t, \bar{a}_{j}-\right)+\eta_{j}(t) S\left(t, \bar{a}_{j}-\right) & & \text { [vaccinated individuals are immunized] }
\end{array}
$$

for a.e. $t>0$ and for every $j \in\{1, \cdots, N\}$. Whenever vaccinations can be dosed only to susceptible individuals, the total cost of the vaccination campaign (1.2) at all ages $\bar{a}_{1}, \ldots, \bar{a}_{N}$ is proportional to the total number of vaccinations dosed, say

$$
\mathcal{C}(\eta)=\sum_{i=1}^{N} \int_{\mathbb{I}} \eta_{i}(t) S\left(t, \bar{a}_{i}-\right) \mathrm{d} t
$$

II being the time interval under consideration and $S$ depending on $\eta$ through (1.2). However, it is reasonable to consider also the case of vaccinations dosed to the $\eta_{j}(t)$ portion of the whole population at time $t$, that is also to infected and immune individuals, in which case (1.3) has to be substituted by

$$
\mathcal{C}(\eta)=\sum_{i=1}^{N} \int_{\mathbb{I}} \eta_{i}(t)\left(S\left(t, \bar{a}_{i}-\right)+I\left(t, \bar{a}_{i}-\right)+R\left(t, \bar{a}_{i}-\right)\right) \mathrm{d} t,
$$

where $S, I$ and $R$ depend on $\eta$ through (1.2). Indeed, not always individuals belonging to the $R$ or even $I$ population can be easily distinguished from those in the $S$ population. Remark that in both cases (1.3) and (1.4), the dynamics of the disease is described by (1.1)-(1.2), since vaccination is assumed to have no effects on $R$ or $I$ individuals.

Alternatively, in a second policy, a vaccination campaign may aim at immunizing an age dependent portion, say $\nu_{1}(a), \ldots, \nu_{N}(a)$, of the $S$ population at given times $\bar{t}_{1}, \ldots, \bar{t}_{N}$. This amounts to substitute (1.2) with

$$
\begin{aligned}
S\left(\bar{t}_{k}+, a\right) & =\left(1-\nu_{k}(a)\right) S\left(\bar{t}_{k}-, a\right) & {\left[\forall a, S\left(\bar{t}_{k}, a\right)\right. \text { decreases due to vaccination] }} \\
I\left(\bar{t}_{k}+, a\right) & =I\left(\bar{t}_{k}-, a\right) & \text { [the infected population is unaltered] } \\
R\left(\bar{t}_{k}+, a\right) & =R\left(\bar{t}_{k}-, a\right)+\nu_{k}(a) S\left(\bar{t}_{k}-, a\right) & \text { [vaccinated individuals are immunized]. }
\end{aligned}
$$

Now, a reasonable cost due to this campaign is

$$
\mathcal{C}(\nu)=\sum_{k=1}^{N} \int_{\mathbb{R}^{+}} \nu_{k}(a) S\left(\bar{t}_{k}-, a\right) \mathrm{d} a
$$


whenever vaccination is dosed only to susceptible individuals. On the other hand, vaccinations can be dosed to all individuals, in which case we replace (1.6) with

$$
\mathcal{C}(\nu)=\sum_{k=1}^{N} \int_{\mathbb{R}^{+}} \nu_{k}(a)\left(S\left(\bar{t}_{k}-, a\right)+I\left(\bar{t}_{k}-, a\right)+R\left(\bar{t}_{k}-, a\right)\right) \mathrm{d} a .
$$

As above, in both cases (1.6) and (1.7), the dynamics of the disease is described by (1.1)-(1.5), since vaccination is assumed to have no effects on $R$ or $I$ individuals.

The most natural way to evaluate the effect of a vaccination campaign is to compute the, possibly weighted, number of infected individuals, namely

$$
\mathcal{E}=\int_{\mathbb{I}} \int_{\mathbb{R}^{+}} \varphi(t, a) I(t, a) \mathrm{d} a \mathrm{~d} t
$$

$\mathcal{E}$ being a function of $\eta$ in case (1.2) and a function of $\nu$ in case (1.5). The dependence of the weight $\varphi$ on time $t$ may account for a possible targeting a decrease in the total number of infected individuals after an initial period, while the dependence of $\varphi$ on $a$ may account for different degrees of danger of the disease at the different ages.

Once the $\operatorname{cost} \mathcal{C}$ and the effect $\mathcal{E}$ are selected, we are left with two modeling choices: "The optimization problem in this framework is to find the strategy with minimal costs at a given level for the effect or to find the strategy with the best effect at given costs.", from [13, Introduction]. In more formal terms, we are lead to tackle the problems

$$
\text { minimize } \mathcal{C} \text { subject to } \mathcal{E} \leq \mathcal{E}_{*} \quad \text { or } \quad \text { minimize } \mathcal{E} \text { subject to } \mathcal{C} \leq \mathcal{C}_{*}
$$

for assigned positive $\mathcal{E}_{*}$ and $\mathcal{C}_{*}$, with time dependent controls $\eta_{i}$ in cases (1.3) or (1.4), or else with age dependent controls $\nu_{k}$ in cases (1.6) or (1.7). The analytic results presented below provide a framework, consisting of well posedness results and stability estimates, where these problems can be rigorously addressed, see [6] for soem specific examples.

The current literature offers a variety of alternative approaches to similar modeling situations. For instance, in the recent [7, the vaccination control enters an equation for $S$ similar to that in (1.1) through a term $-u S$ in the right hand side, meaning that vaccination takes place uniformly at all ages. A similar approach is followed also in [12, 13].

From the analytic point of view, below we prove well posedness and stability for (1.1)-(1.2) and for (1.1)-(1.5) which, in turn, ensure the existence of optimal vaccination strategies. To achieve this, we prove well posedness and stability of a more general IBVP, see (3.1).

The next section presents solutions to problems (1.9), as a consequence of the analytic framework developed in Section 3, All analytic proofs are collected in Section 4.

\section{The Controlled SIR Models}

Denote by $\mathbb{I}$ the time interval $[0, T]$, for a positive $T$, or $[0,+\infty[$.

Throughout, we supplement (1.1) with the initial and boundary conditions

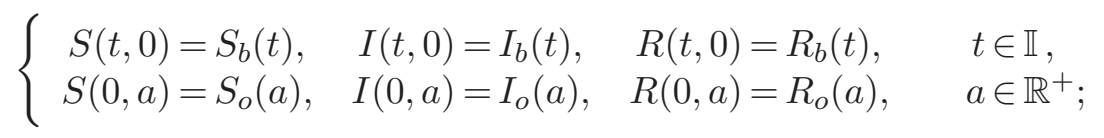


We require below the following assumptions on the functions defining (1.1)-(2.1) 1 :

( $\lambda) \quad \lambda \in \mathbf{C}^{\mathbf{0}}\left(\mathbb{R}^{+} \times \mathbb{R}^{+} ; \mathbb{R}\right)$ admits positive constants $\Lambda_{\infty}, \Lambda_{L}$ such that for all $a_{1}, a_{2}, a^{\prime} \in \mathbb{R}^{+}$

$$
\begin{aligned}
\|\lambda\|_{\mathbf{L}^{\infty}\left(\mathbb{R}^{+} \times \mathbb{R}^{+} ; \mathbb{R}\right)}+\operatorname{TV}\left(\lambda\left(\cdot, a^{\prime}\right) ; \mathbb{R}^{+}\right) & \leq \Lambda_{\infty} \\
\left|\lambda\left(a_{1}, a^{\prime}\right)-\lambda\left(a_{2}, a^{\prime}\right)\right| & \leq \Lambda_{L}\left|a_{1}-a_{2}\right| .
\end{aligned}
$$

(dr) The maps $d_{S}, d_{I}, d_{R}, r_{I}: \mathbb{I} \times \mathbb{R}^{+} \rightarrow \mathbb{R}$ are Caratheodory functions, in the sense of Definition 4.2, and there exist positive $R_{L}, R_{1}, R_{\infty}$ such that for $\varphi=d_{S}, d_{I}, d_{R}, r_{I}, t \in \mathbb{I}$ and $a_{1}, a_{2} \in \mathbb{R}^{+}$,

$$
\begin{aligned}
\|\varphi\|_{\mathbf{L}^{\infty}\left(\mathbb{I} \times \mathbb{R}^{+} ; \mathbb{R}\right)}+\operatorname{TV}\left(\varphi(t, \cdot) ; \mathbb{R}^{+}\right) & \leq R_{\infty} \\
\left|\varphi\left(t, a_{2}\right)-\varphi\left(t, a_{1}\right)\right| & \leq R_{L}\left|a_{2}-a_{1}\right| \\
\|\varphi\|_{\mathbf{C}^{\mathbf{0}}\left(\mathbb{I} ; \mathbf{L}^{1}\left(\mathbb{R}^{+} ; \mathbb{R}\right)\right)} & \leq R_{1} .
\end{aligned}
$$

(IB) The initial and boundary data satisfy

$$
S_{o}, I_{o}, R_{o} \in\left(\mathbf{L}^{\mathbf{1}} \cap \mathbf{B V}\right)\left(\mathbb{R}^{+} ; \mathbb{R}^{+}\right) \quad \text { and } \quad S_{b}, I_{b}, R_{b} \in\left(\mathbf{L}^{\mathbf{1}} \cap \mathbf{B V}\right)\left(\mathbb{I} ; \mathbb{R}^{+}\right) .
$$

First, we provide the basic well posedness result for the model presented above, based on the nonlocal renewal equations (1.1), in the case of the vaccination policy (1.2).

Theorem 2.1. Under hypotheses $[\boldsymbol{( \lambda )}$ and $[(\boldsymbol{d r})$, for any initial and boundary data satisfying $\left[(\boldsymbol{I B})\right.$, for any choice of the vaccination ages $\bar{a}_{1}, \ldots, \bar{a}_{N}$ and of the control function $\eta \in \mathbf{B V}\left(\mathbb{I} ;[0,1]^{N}\right)$, problem (1.1)-(1.2)-(2.1) admits a unique solution

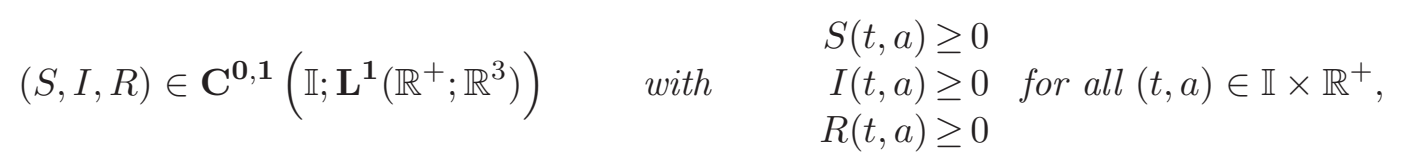

depending Lipschitz continuously on the initial datum, through its $\mathbf{L}^{\mathbf{1}}$ norm, and on $\eta$, through its $\mathbf{L}^{\infty}$ norm.

The proof, deferred to $\S$ 4.4, amounts to show that problem (1.1)-(1.2)-(2.1) satisfies the assumptions of Theorem 3.1 and of Corollary 3.3 below.

In the case of the vaccination policy (1.5), we obtain an analogous result.

Theorem 2.2. Under hypotheses $[\boldsymbol{( \lambda )}$ and $[(\boldsymbol{d r})$, for any initial and boundary data satisfying $(\mathbf{I B})$, for any choice of the vaccination times $\bar{t}_{1}, \ldots, \bar{t}_{N}$ and of the control function $\nu \in \mathbf{B V}\left(\mathbb{I} ;[0,1]^{N}\right)$, problem (1.1) -(1.5) -(2.1) admits a unique solution as in (2.8), depending Lipschitz continuously on the initial datum, through the $\mathbf{L}^{\mathbf{1}}$ norm, and on $\nu$, through the $\mathbf{L}^{\infty}$ norm.

The proof is deferred to $\S 4.4$.

Once Theorem 2.1 and Theorem 2.2 are acquired, both $\operatorname{costs} \mathcal{C}$ and $\mathcal{E}$ are easily shown to be strongly continuous functions of the control $\eta$. The existence of an optimal strategy then follows through an application of Weierstraß Theorem, as soon as the choice of $\eta$ or $\nu$ is restricted to a suitable strongly compact set. We refer to [6] for a selection of control problems based on Theorem 2.1 or Theorem 2.2.

\footnotetext{
${ }^{1}$ Throughout, we strive to have dimensionally correct expressions at the cost of distinguishing the various constants whenever they are dimensionally different.
} 


\section{Analytic Results}

The proofs of Theorem 2.1 and of Theorem 2.2 follow from a slightly more general statement.

Theorem 3.1. Consider the following Initial - Boundary Value Problem (IBVP)

$$
\left\{\begin{array}{l}
\partial_{t} u_{i}+\partial_{x}\left(g_{i}(t, x) u_{i}\right)=\left(\alpha_{i}[u(t)](x)+\gamma_{i}(t, x)\right) \cdot u \\
u_{i}(0, x)=u_{o, i}(x) \\
g_{i}(t, 0+) u_{i}(t, 0+)=\beta_{i}\left(t, u_{1}\left(t, \bar{x}_{1}-\right), \ldots, u_{n}\left(t, \bar{x}_{n}-\right)\right)
\end{array} \quad i=1, \ldots, n\right.
$$

where

(IBVP.1) $g_{1}, \cdots, g_{n} \in \mathbf{C}^{\mathbf{0}, \mathbf{1}}\left(\mathbb{I} \times \mathbb{R}^{+} ;[\check{g}, \hat{g}]\right)$ and for all $t \in \mathbb{I}, x \in \mathbb{R}^{+}, i=1, \ldots, n$

$$
\begin{aligned}
\operatorname{TV}\left(g_{i}(\cdot, x) ; \mathbb{I}\right)+\operatorname{TV}\left(g_{i}(t, \cdot) ; \mathbb{R}^{+}\right) & \leq G_{\infty} \\
\operatorname{TV}\left(\partial_{x} g_{i}(t, \cdot) ; \mathbb{R}^{+}\right)+\left\|\partial_{x} g_{i}(t, \cdot)\right\|_{\mathbf{L}^{\infty}\left(\mathbb{R}^{+} ; \mathbb{R}\right)} & \leq G_{1} .
\end{aligned}
$$

(IBVP.2) $\alpha_{1}, \ldots, \alpha_{n}: \mathbf{L}^{\mathbf{1}}\left(\mathbb{R}^{+} ; \mathbb{R}^{n}\right) \rightarrow \mathbf{C}^{\mathbf{0}}\left(\mathbb{R}^{+} ; \mathbb{R}^{n}\right)$ are linear and continuous maps and there exist positive constants $A_{L}$ and $A_{1}$ such that

$$
\begin{aligned}
& \left\|\alpha_{i}[w]\right\|_{\mathbf{L}^{\infty}\left(\mathbb{R}^{+} ; \mathbb{R}^{n}\right)}+\operatorname{TV}\left(\alpha_{i}[w] ; \mathbb{R}^{+}\right) \leq A_{L}\|w\|_{\mathbf{L}^{\mathbf{1}}\left(\mathbb{R}^{+} ; \mathbb{R}^{n}\right)}
\end{aligned}
$$

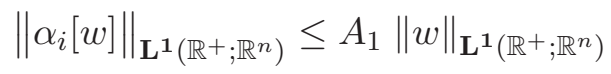

for every $i \in\{1, \cdots, n\}, w \in \mathbf{L}^{\mathbf{1}}\left(\mathbb{R}^{+} ; \mathbb{R}^{n}\right)$. Moreover, for every $w \in\left(\mathbf{L}^{\mathbf{1}} \cap \mathbf{L}^{\infty}\right)\left(\mathbb{R}^{+} ; \mathbb{R}\right)$, there exists a positive $A_{2}$ such that for all $x_{1}, x_{2} \in \mathbb{R}^{+}$

$$
\left\|\alpha_{i}[w]\left(x_{1}\right)-\alpha_{i}[w]\left(x_{2}\right)\right\| \leq A_{2}\left|x_{1}-x_{2}\right| .
$$

(IBVP.3) $\gamma_{1}, \ldots, \gamma_{n} \in \mathbf{C}^{\mathbf{0}}\left(\mathbb{I} ; \mathbf{L}^{\mathbf{1}}\left(\mathbb{R}^{+} ; \mathbb{R}^{n}\right)\right)$ are Caratheodory functions, in the sense of Definition 4.2, and there exist positive constants $C_{L}, C_{\infty}$ such that

$$
\begin{aligned}
\left\|\gamma_{i}\left(t, x_{2}\right)-\gamma_{i}\left(t, x_{1}\right)\right\| & \leq C_{L}\left|x_{2}-x_{1}\right| \\
\left\|\gamma_{i}(t, \cdot)\right\|_{\mathbf{L}^{\infty}\left(\mathbb{R}^{+} ; \mathbb{R}\right)}+\operatorname{TV}\left(\gamma_{i}(t, \cdot) ; \mathbb{R}^{+}\right) & \leq C_{\infty}
\end{aligned}
$$

for every $i \in\{1, \cdots, n\}, t \in \mathbb{I}$ and $x_{1}, x_{2} \in \mathbb{R}^{+}$.

(IBVP.4) $\beta_{1}, \ldots, \beta_{n}$ are Caratheodory functions in the sense of Definition 4.2. for all $t$, $\beta_{1}(t), \ldots, \beta_{n}(t) \in \mathbf{C}^{\mathbf{1}}\left(\mathbb{R}^{n} ; \mathbb{R}\right)$ and, for all $(t, u) \in \mathbb{I} \times \mathbb{R}^{n}, \partial_{u_{j}} \beta_{i}(t, u)=0$ whenever $j \geq i$. Moreover, there exist constants $B_{1}, B_{\infty}$ and $B_{L}$ such that

$$
\begin{aligned}
\left|\beta_{i}\left(t, u_{1}\right)-\beta_{i}\left(t, u_{2}\right)\right| & \leq B_{L}\left\|u_{1}-u_{2}\right\| \\
\left\|\beta_{i}(\cdot, 0)\right\|_{\mathbf{L}^{1}(\mathbb{I} ; \mathbb{R})} & \leq B_{1} \\
\left\|\beta_{i}(\cdot, 0)\right\|_{\mathbf{L}^{\infty}(\mathbb{I} ; \mathbb{R})} & \leq B_{\infty} \\
\operatorname{TV}\left(\beta_{i}(\cdot, u(\cdot)) ; \mathbb{I}\right) & \leq B_{\infty}+B_{L} \operatorname{TV}(u ; \mathbb{I})
\end{aligned}
$$

for every $i \in\{1, \cdots, n\}, t \in \mathbb{I} u_{1}, u_{2} \in \mathbb{R}^{n}$ and $u \in \mathbf{B V}\left(\mathbb{I} ; \mathbb{R}^{n}\right)$.

(IBVP.5) $u_{o} \in \mathbf{L}^{1}\left(\mathbb{R}^{+} ; \mathbb{R}^{n}\right)$. 
Then, there exists constants $K_{1}$ and $K_{\infty}$, a positive time $t_{*}$ and a constant $\mathcal{L}$ dependent only on $t_{*},\left\|u_{o}\right\|_{\mathbf{L}^{1}\left(\mathbb{R}^{+} ; \mathbb{R}^{n}\right)}, \operatorname{TV}\left(u^{o} ; \mathbb{R}^{+}\right)$and on the parameters in (IBVP.1) (IBVP.5) such that (3.1) admits a unique solution

$$
u_{*} \in \mathbf{C}^{\mathbf{0}}\left(\left[0, t_{*}\right] ; \mathbf{L}^{\mathbf{1}}\left(\mathbb{R}^{+} ; \mathbb{R}^{n}\right)\right) \text {, }
$$

satisfying, for all $t, t^{\prime} \in\left[0, t_{*}\right]$,

$$
\begin{aligned}
& \left\|u_{*}(t)\right\|_{\mathbf{L}^{\mathbf{1}\left(\mathbb{R}^{+} ; \mathbb{R}^{n}\right)}} \leq K_{1}, \quad\left\|u_{*}(t)-u_{*}\left(t^{\prime}\right)\right\|_{\mathbf{L}^{\mathbf{1}}\left(\mathbb{R}^{+} ; \mathbb{R}^{n}\right)} \leq \mathcal{L}\left|t-t^{\prime}\right|, \\
& \left\|u_{*}(t)\right\|_{\mathbf{L}^{\infty}\left(\mathbb{R}^{+} ; \mathbb{R}^{n}\right)} \leq K_{\infty}, \quad \operatorname{TV}\left(u_{*}(t) ; \mathbb{R}^{+}\right) \leq K_{\infty} .
\end{aligned}
$$

Moreover, if $u_{*}^{\prime}$ and $u_{*}^{\prime \prime}$ are the solutions to (3.1) corresponding to initial data $u_{o}^{\prime}$ and $u_{o}^{\prime \prime}$ and to boundary data $\beta^{\prime}$ and $\beta^{\prime \prime}$, then the following estimate holds for all $t \in\left[0, t_{*}\right]$ :

$$
\left\|u_{*}^{\prime}(t)-u_{*}^{\prime \prime}(t)\right\|_{\mathbf{L}^{1}\left(\mathbb{R}^{+} ; \mathbb{R}^{n}\right)} \leq \mathcal{L}\left(\left\|u_{o}^{\prime}-u_{o}^{\prime \prime}\right\|_{\mathbf{L}^{1}\left(\mathbb{R}^{+} ; \mathbb{R}^{n}\right)}+\left\|\beta^{\prime}-\beta^{\prime \prime}\right\|_{\mathbf{L}^{\infty}\left(\left[0, t_{*}\right] \times\left[0, K_{\infty}\right]^{n} ; \mathbb{R}^{n}\right)}\right) .
$$

The proof is detailed in $\S 4.3$. In particular, lower bounds for $K_{1}$ and $K_{\infty}$ are in (4.29). Above, solutions to (3.1) are intended essentially in the sense of Definition 4.3. note indeed that for each $i=1, \ldots, n$, problem (3.1) fits into (4.8), refer to (4.32) for the details.

Remark 3.2. The assumptions in Theorem 3.1 do not rule out a finite time blow-up in u. In fact, consider the IBVP

$$
\left\{\begin{array}{l}
\partial_{t} u+\partial_{x} u=\int_{\mathbb{R}^{+}} u(t, \xi) \mathrm{d} \xi u \\
u(0, x)=e^{-x} \\
u(t, 0)=0
\end{array}\right.
$$

that clearly fits into (IBVP.1) (IBVP.5) with $\alpha[w]=\int_{\mathbb{R}^{+}} w(\xi) \mathrm{d} \xi$ and $\mathbb{I}=\mathbb{R}^{+}$. Its (strong) solution for $(t, x) \in\left[0, \ln 2\left[\times \mathbb{R}^{+}\right.\right.$is $u(t, x)=\frac{e^{t-x}}{2-e^{t}}$, which blows up as $t \rightarrow \ln 2$.

Motivated by Remark 3.2. we now strengthen the assumptions in Theorem 3.1 to ensure two properties of key interest in the vaccination model (1.1)-(2.1), namely that the solution $u$ attains positive values and that it is defined on all $\mathbb{I}$.

Corollary 3.3. Assume that, besides all assumptions (IBVP.1) (IBVP.5) in Theorem 3.1, we also have that

(POS) For all $i, u_{i}^{o} \geq 0$ and $\beta_{i} \geq 0$.

(NEG) For all $u \in \mathbf{L}^{\mathbf{1}}\left(\mathbb{R}^{+} ;\left(\mathbb{R}^{+}\right)^{n}\right), t \in \mathbb{I}$ and $x \in \mathbb{R}^{+}, \sum_{i=1}^{n}\left(\alpha_{i}[u](x)+\gamma_{i}(t, x)\right) \cdot u(x) \leq 0$.

(EQ) For all $i, j=1, \ldots, n, t \in \mathbb{I}$ and $x \in \mathbb{R}^{+}, g_{i}(t, x)=g_{j}(t, x)$.

Then, each component of the solution $u_{*}$ constructed in Theorem 3.1 attains non negative values. Moreover, $u_{*}$ can be uniquely extended to all $\mathbb{I}$.

The proof is deferred to $\S 4.3$,

\section{Proofs}

Let $J$ denote a (non empty) real interval. We use throughout the norms

$$
\begin{array}{rlrl}
\|f\|_{\mathbf{C}^{0}\left(J ; \mathbf{L}^{1}\left(\mathbb{R}^{+} ; \mathbb{R}\right)\right)} & =\sup _{t \in J}\|f\|_{\mathbf{L}^{1}\left(\mathbb{R}^{+} ; \mathbb{R}\right)} ; & \|f\|_{\mathbf{L}^{1}\left(\mathbb{R}^{+} ; \mathbb{R}\right)} & =\int_{\mathbb{R}^{+}}|f(x)| \mathrm{d} x \\
\|f\|_{\mathbf{C}^{\mathbf{0}}\left(J ; \mathbf{L}^{\infty}\left(\mathbb{R}^{+} ; \mathbb{R}\right)\right)} & =\sup _{t \in J}\|f\|_{\mathbf{L}^{\infty}\left(\mathbb{R}^{+} ; \mathbb{R}\right)} ; & \|f\|_{\mathbf{L}^{\infty}\left(\mathbb{R}^{+} ; \mathbb{R}\right)} & =\operatorname{esss}_{x \in \mathbb{R}^{+}}|f(x)| ; \\
\|f\|_{\mathbf{L}^{1}\left(\mathbb{R}^{+} ; \mathbb{R}^{n}\right)} & =\sum_{i=1}^{n}\left\|f_{i}\right\|_{\mathbf{L}^{1}(\mathbb{R}+; \mathbb{R})}
\end{array}
$$




\subsection{Preliminary Properties of BV Functions}

Recall the following elementary estimates on BV functions, see also [5, Section 4] or [1]:

$$
\begin{aligned}
& \left.\begin{array}{r}
u \in \mathbf{B V}\left(\mathbb{R}^{+} ; \mathbb{R}\right) \\
w \in \mathbf{B V}\left(\mathbb{R}^{+} ; \mathbb{R}\right)
\end{array}\right\} \Rightarrow \operatorname{TV}(u w) \leq \mathrm{TV}(u)\|w\|_{\mathbf{L}^{\infty}\left(\mathbb{R}^{+} ; \mathbb{R}\right)}+\|u\|_{\mathbf{L}^{\infty}\left(\mathbb{R}^{+} ; \mathbb{R}\right)} \operatorname{TV}(w) ; \\
& \left.\begin{array}{l}
\varphi \in \mathbf{C}^{\mathbf{0}, \mathbf{1}}\left(\mathbb{R}^{n} ; \mathbb{R}\right) \\
u \in \mathbf{B V}\left(\mathbb{R}^{+} ; \mathbb{R}^{n}\right)
\end{array}\right\} \Rightarrow \operatorname{TV}(\varphi \circ u) \leq \operatorname{Lip}(\varphi) \mathrm{TV}(u) \\
& \left.\begin{array}{rl}
u & \in \mathbf{B V}\left(\mathbb{R}^{+} ; \mathbb{R}\right) \\
w & \in \mathbf{B V}\left(\mathbb{R}^{+} ; \mathbb{R}\right) \\
(x) & \geq \check{w}>0
\end{array}\right\} \Rightarrow \operatorname{TV}\left(\frac{u}{w}\right) \leq \frac{1}{\check{w}} \operatorname{TV}(u)+\frac{1}{\check{w}^{2}} \operatorname{TV}(w)\|u\|_{\mathbf{L}^{\infty}\left(\mathbb{R}^{+} ; \mathbb{R}\right)} ; \\
& \left.\begin{array}{l}
u \in \mathbf{L}^{\mathbf{1}}\left(J ; \mathbf{L}^{\mathbf{1}}\left(\mathbb{R}^{+} ; \mathbb{R}\right)\right) \\
u(t) \in \mathbf{B V}\left(\mathbb{R}^{+} ; \mathbb{R}\right)
\end{array}\right\} \Rightarrow \operatorname{TV}\left(\int_{0}^{t} u(\tau, \cdot) \mathrm{d} \tau\right) \leq \int_{0}^{t} \mathrm{TV}(u(\tau)) \mathrm{d} \tau \\
& \left.\begin{array}{l}
u \in \mathbf{B V}\left(\mathbb{R}^{+} ; \mathbb{R}\right) \\
\delta \in \mathbf{L}^{\infty}\left(\mathbb{R} ; \mathbb{R}^{+}\right)
\end{array}\right\} \Rightarrow \int_{\mathbb{R}^{+}}|u(x+\delta(x))-u(x)| \mathrm{d} x \leq \mathrm{TV}(u)\|\delta\|_{\mathbf{L}^{\infty}\left(\mathbb{R}^{+} ; \mathbb{R}\right)} .
\end{aligned}
$$

Inequality (4.2) follows from [1, Formula (3.10)]. The definition of total variation directly implies (4.3), (4.4) and (4.5). For a proof of (4.6) see for instance [3, Lemma 2.3]. We supplement the estimates above with the following one.

Lemma 4.1. Let $J=\left[0, t_{*}\right]$, with $t_{*}>0$. Assume that $u \in \mathbf{L}^{\infty}(J \times J ; \mathbb{R})$ is such that $\sup _{\tau \in J} \operatorname{TV}(u(\tau, \cdot) ; J)<+\infty$. Then, setting $U(t)=\int_{0}^{t} u(\tau, t) \mathrm{d} \tau$,

$$
\operatorname{TV}(U ; J) \leq\|u\|_{\mathbf{L}^{\infty}(J \times J ; \mathbb{R})} t_{*}+\int_{J} \operatorname{TV} u(\tau, \cdot) \mathrm{d} \tau
$$

Proof. Fix $t_{0}, t_{1}, \ldots, t_{n}$ in $J$ with $t_{i-1}<t_{i}$ for $i=1, \ldots, n$. Using [1, Theorem 3.27 and (3.24)],

$$
\begin{aligned}
\sum_{i=1}^{n}\left|U\left(t_{i}\right)-U\left(t_{i-1}\right)\right| & =\sum_{i=1}^{n}\left|\int_{0}^{t_{i}} u\left(\tau, t_{i}\right) \mathrm{d} \tau-\int_{0}^{t_{i-1}} u\left(\tau, t_{i-1}\right) \mathrm{d} \tau\right| \\
& =\sum_{i=1}^{n}\left|\int_{t_{i-1}}^{t_{i}} u\left(\tau, t_{i}\right) \mathrm{d} \tau+\int_{0}^{t_{i-1}}\left(u\left(\tau, t_{i}\right)-u\left(\tau, t_{i-1}\right)\right) \mathrm{d} \tau\right| \\
& \leq \sum_{i=1}^{n} \int_{t_{i-1}}^{t_{i}}\left|u\left(\tau, t_{i}\right)\right| \mathrm{d} \tau+\sum_{i=1}^{n} \int_{0}^{t_{i-1}}\left|u\left(\tau, t_{i}\right)-u\left(\tau, t_{i-1}\right)\right| \mathrm{d} \tau \\
& \leq \sum_{i=1}^{n} \int_{t_{i-1}}^{t_{i}}\|u\|_{\mathbf{L}^{\infty}(J \times J ; \mathbb{R})} \mathrm{d} \tau+\sum_{i=1}^{n} \int_{J}\left|u\left(\tau, t_{i}\right)-u\left(\tau, t_{i-1}\right)\right| \mathrm{d} \tau \\
& \leq\|u\|_{\mathbf{L}^{\infty}(J \times J ; \mathbb{R})} t_{*}+\int_{J} \mathrm{TV} u(\tau, \cdot) \mathrm{d} \tau
\end{aligned}
$$

which completes the proof. 


\subsection{A Scalar Renewal Equation}

We consider the following initial-boundary value problem for a linear scalar balance law, or renewal equation, see also [15, Chapter 3], of the form

$$
\left\{\begin{array}{lc}
\partial_{t} u+\partial_{x}(g(t, x) u)+m(t, x) u=f(t, x) & (t, x) \in J \times \mathbb{R}^{+}, \\
u(0, x)=u_{o}(x) & x \in \mathbb{R}^{+}, \\
g(t, 0) u(t, 0+)=b(t) & t \in J
\end{array}\right.
$$

Let $F_{1}, F_{\infty}, G_{1}, G_{\infty}, M, \hat{g}, \check{g}$ be positive with $\check{g}<\hat{g}$. We require the following conditions:

(f) $\quad f \in \mathbf{C}^{\mathbf{0}}\left(J ; \mathbf{L}^{\mathbf{1}}\left(\mathbb{R}^{+} ; \mathbb{R}\right)\right)$ and for all $t \in J\left\{\begin{array}{r}\|f(t, \cdot)\|_{\mathbf{L}^{1}\left(\mathbb{R}^{+} ; \mathbb{R}\right)} \leq F_{1} ; \\ \|f(t, \cdot)\|_{\mathbf{L}^{\infty}\left(\mathbb{R}^{+} ; \mathbb{R}\right)}+\operatorname{TV}\left(f(t, \cdot) ; \mathbb{R}^{+}\right) \leq F_{\infty}\end{array}\right.$

(g) $g \in \mathbf{C}^{\mathbf{0}, \mathbf{1}}\left(J \times \mathbb{R}^{+} ;[\check{g}, \hat{g}]\right)$, for $(t, x) \in J \times \mathbb{R}^{+}\left\{\begin{array}{r}\operatorname{TV}\left(g(t, \cdot) ; \mathbb{R}^{+}\right)+\operatorname{TV}(g(\cdot, x) ; J) \leq G_{\infty} ; \\ \left\|\partial_{x} g(t, \cdot)\right\|_{\mathbf{L}^{\infty}\left(\mathbb{R}^{+} ; \mathbb{R}\right)}+\operatorname{TV}\left(\partial_{x} g(t, \cdot) ; \mathbb{R}^{+}\right) \leq G_{1}\end{array}\right.$

(m) $m$ is a Caratheodory function and for all $t \in J,\|m(t, \cdot)\|_{\mathbf{L}^{\infty}\left(\mathbb{R}^{+} ; \mathbb{R}\right)}+\mathrm{TV}\left(m(t, \cdot) ; \mathbb{R}^{+}\right) \leq M$;

(b) $\quad b \in \mathbf{B V}_{\text {loc }}(J ; \mathbb{R})$.

Above, we refer to the usual definition of Caratheodory function, namely:

Definition 4.2 ([4, (A) and (B) in $\S 3.1]$ ). The map $m: J \times \mathbb{R}^{+} \rightarrow \mathbb{R}^{m}$ is a Caratheodory function if

1. For all $x \in \mathbb{R}^{+}$, the map $m_{x}: J \rightarrow \mathbb{R}^{m}$ defined by $m_{x}(t)=m(t, x)$ is measurable.

2. For a.e. $t \in J$, the map $m^{t}: \mathbb{R}^{+} \rightarrow \mathbb{R}^{m}$ defined by $m^{t}(x)=m(t, x)$ is continuous.

3. For all compact $K \subset J \times \mathbb{R}^{+}$, there exist constants $M_{\infty}, M_{L}>0$ such that for a.e. $t \in J$ and for all $x_{1}, x_{2} \in \mathbb{R}^{+},\|m(t, x)\| \leq M_{\infty}$ and $\left\|m\left(t, x_{2}\right)-m\left(t, x_{1}\right)\right\| \leq M_{L} \cdot\left|x_{2}-x_{1}\right|$.

Recall the following definition of solution to (4.8), see also [2, 3, 11, 15, 16, 17].

Definition 4.3. Assume that $(\mathbf{f})$, (g), $\left(\mathbf{( m )}\right.$ and $\left(\mathbf{( b )}\right.$ hold. Choose an initial datum $u_{o} \in$ $\mathbf{L}^{\mathbf{1}}\left(\mathbb{R}^{+} ; \mathbb{R}\right)$. The function $u \in \mathbf{C}^{0}\left(J ; \mathbf{L}^{1}\left(\mathbb{R}^{+} ; \mathbb{R}\right)\right)$ is a solution to (4.8) if

1. for all $\varphi \in \mathbf{C}_{\mathbf{c}}^{\mathbf{1}}\left(\stackrel{\circ}{J} \times \stackrel{\circ}{\mathbb{R}}^{+} ; \mathbb{R}\right), \int_{\mathbb{R}^{+}} \int_{J}\left[u \partial_{t} \varphi+g u \partial_{x} \varphi+(f-m u) \varphi\right] \mathrm{d} t \mathrm{~d} x=0$;

2. $u(0, x)=u_{o}(x)$ for a.e. $x \in \mathbb{R}^{+}$;

3. for a.e. $t \in J, \lim _{x \rightarrow 0+} g(t, x) u(t, x)=b(t)$.

As shown below, problem (4.8) admits as unique solution in the sense of Definition 4.3 the map

$$
u(t, x)= \begin{cases}u_{o}(X(0 ; t, x)) \mathcal{E}(0, t, x)+\int_{0}^{t} f(\tau, X(\tau ; t, x)) \mathcal{E}(\tau, t, x) \mathrm{d} \tau & x>\sigma(t) \\ \frac{b(T(0 ; t, x))}{g(T(0 ; t, x), 0)} \mathcal{E}(T(0 ; t, x), t, x)+\int_{T(0 ; t, x)}^{t} f(\tau, X(\tau ; t, x)) \mathcal{E}(\tau, t, x) \mathrm{d} \tau & x<\sigma(t)\end{cases}
$$


where

$$
\mathcal{E}(\tau, t, x)=\exp \left[-\int_{\tau}^{t}\left(m(s, X(s ; t, x))+\partial_{x} g(s, X(s ; t, x))\right) \mathrm{d} s\right]
$$

and, for $t_{o}, t \in J, x_{o}, x \in \mathbb{R}^{+}$,

$$
t \rightarrow X\left(t ; t_{o}, x_{o}\right) \text { solves }\left\{\begin{array} { l } 
{ \dot { x } = g ( t , x ) } \\
{ x ( t _ { o } ) = x _ { o } }
\end{array} \text { and } \quad x \rightarrow T ( x ; t _ { o } , x _ { o } ) \text { solves } \left\{\begin{array}{l}
t^{\prime}=\frac{1}{g(t, x)} \\
t\left(x_{o}\right)=t_{o}
\end{array}\right.\right.
$$

and we also set $\sigma(t)=X(t ; 0,0), \Sigma(x)=T(x ; 0,0)$.

Lemma 4.4. Let $(\mathrm{g})$ and $(\mathbf{m})$ hold. Then, $\mathcal{E}$ defined in (4.10) satisfies the following estimates, for $x \in \mathbb{R}^{+}$and $\tau, t \in J$ with $\tau \leq t$ :

$$
\begin{aligned}
\mathcal{E}(\tau, t, x) & \leq e^{\left(G_{1}+M\right)(t-\tau)} \\
\operatorname{TV}\left(\mathcal{E}(\tau, t, \cdot) ; \mathbb{R}^{+}\right) & \leq\left(G_{1}+M\right)(t-\tau) e^{\left(G_{1}+M\right)(t-\tau)} \\
\operatorname{TV}(\mathcal{E}(\tau, \cdot, x) ;[0, t]) & \leq\left(G_{1}+M\right)(t-\tau) e^{\left(G_{1}+M\right)(t-\tau)} \\
\operatorname{TV}(\mathcal{E}(\cdot, t, x) ;[0, t]) & \leq\left(G_{1}+M\right) t e^{\left(G_{1}+M\right) t}
\end{aligned}
$$

Proof. The bound (4.12) directly follows from (g), (m), and (4.10). Consider the total variation estimate (4.13). For $\tau \leq t$, by (4.3) we have

$$
\operatorname{TV}\left(\mathcal{E}(\tau, t, \cdot) ; \mathbb{R}^{+}\right) \leq e^{\left(G_{1}+M\right)(t-\tau)} \int_{\tau}^{t}\left(\operatorname{TV}\left(m(s, \cdot) ; \mathbb{R}^{+}\right)+\operatorname{TV}\left(\partial_{x} g(s, \cdot) ; \mathbb{R}^{+}\right)\right) \mathrm{d} s
$$

which implies (4.13). Now, consider the total variation estimate (4.14). For $\tau \leq t$ we deduce

$$
\begin{aligned}
& \operatorname{TV}(\mathcal{E}(\tau, \cdot, x) ;[0, t]) \\
\leq & e^{\left(G_{1}+M\right)(t-\tau)} \operatorname{TV}\left(\int_{\tau}\left[m(s, X(s ; \cdot, x))+\partial_{x} g(s, X(s ; \cdot, x))\right] \mathrm{d} s\right) \\
\leq & e^{\left(G_{1}+M\right)(t-\tau)}\left[\|m\|_{\mathbf{L}^{\infty}\left([0, t] \times \mathbb{R}^{+} ; \mathbb{R}\right)}+\left\|\partial_{x} g\right\|_{\mathbf{L}^{\infty}\left([0, t] \times \mathbb{R}^{+} ; \mathbb{R}\right)}\right](t-\tau) \\
& +e^{\left(G_{1}+M\right)(t-\tau)}\left[\sup _{s \in[0, t]} \operatorname{TV}\left(m(s, X(s ; \cdot, x))+\partial_{x} g(s, X(s ; \cdot, x))\right)\right](t-\tau)
\end{aligned}
$$

and using (g) and (m) we deduce (4.14). Finally, consider the estimate (4.15). We have

$$
\begin{aligned}
& \operatorname{TV}(\mathcal{E}(\cdot, t, x) ;[0, t]) \\
\leq & e^{\left(G_{1}+M\right) t} \operatorname{TV}\left(\int^{t}\left[m(s, X(s ; t, x))+\partial_{x} g(s, X(s ; t, x))\right] \mathrm{d} s\right) \quad \text { [by (44.3)] } \\
\leq & e^{\left(G_{1}+M\right) t}\left[\|m\|_{\mathbf{L}^{\infty}\left([0, t] \times \mathbb{R}^{+} ; \mathbb{R}\right)}+\left\|\partial_{x} g\right\|_{\mathbf{L}^{\infty}\left([0, t] \times \mathbb{R}^{+} ; \mathbb{R}\right)}\right] t,
\end{aligned}
$$

concluding the proof.

The following Lemma summarizes various properties of the solution to (4.8), see also [15]. 
Lemma 4.5. Let (f), (g) and (m) hold. Then, with reference to the scalar problem (4.8),

(SP.1) For any $u_{o} \in\left(\mathbf{L}^{\mathbf{1}} \cap \mathbf{B V}\right)\left(\mathbb{R}^{+} ; \mathbb{R}\right)$ and for any $b$ satisfying $(\mathbf{b})$, the map $u: J \times \mathbb{R}^{+} \rightarrow$ $\mathbb{R}$ defined by (4.9) solves (4.8) in the sense of Definition 4.3 .

(SP.2) For every $t \in J$, the following a priori estimates hold:

$$
\begin{aligned}
\sup _{\tau \in[0, t]}\|u(\tau)\|_{\mathbf{L}^{\infty}(\mathbb{R} ; ; \mathbb{R})} \leq\left(\left\|u_{o}\right\|_{\mathbf{L}^{\infty}\left(\mathbb{R}^{+} ; \mathbb{R}\right)}+\frac{1}{\check{g}}\|b\|_{\mathbf{L}^{\infty}([0, t] ; \mathbb{R})}+F_{\infty} t\right) e^{\left(G_{1}+M\right) t}, \\
\sup _{\tau \in[0, t]}\|u(\tau)\|_{\mathbf{L}^{1}\left(\mathbb{R}^{+} ; \mathbb{R}\right)} \leq\left(\left\|u_{o}\right\|_{\mathbf{L}^{1}\left(\mathbb{R}^{+} ; \mathbb{R}\right)}+\|b\|_{\mathbf{L}^{\mathbf{1}([0, t] ; \mathbb{R})}}+F_{1} t\right) e^{M t} .
\end{aligned}
$$

(SP.3) For every $t \in J$, the following total variation estimate holds

$$
\operatorname{TV}\left(u(t) ; \mathbb{R}^{+}\right) \leq \mathcal{H}(t)\left(F_{\infty} t+\frac{\|b\|_{\mathbf{L}^{\infty}([0, t] ; \mathbb{R})}+\mathrm{TV}(b ;[0, t])}{\check{g}}+\left\|u_{o}\right\|_{\mathbf{L}^{\infty}\left(\mathbb{R}^{+} ; \mathbb{R}\right)}+\mathrm{TV}\left(u_{o} ; \mathbb{R}^{+}\right)\right)
$$

where $\mathcal{H}(t)$ is a non decreasing continuous function of $t$, depending also on $\check{g}, G_{1}, G_{\infty}$ and $M$, satisfying $\mathcal{H}(0) \leq 5+G_{\infty} / \check{g}$.

(SP.4) Fix $t \in J$ and $x \in \mathbb{R}^{+}$. If $x>\sigma(t)$, then

$$
\begin{aligned}
\operatorname{TV}(u(\cdot, x) ;[0, t]) \leq[ & \left.\operatorname{TV}\left(u_{o} ; \mathbb{R}^{+}\right)+2\left(G_{1}+M\right)\left\|u_{o}\right\|_{\mathbf{L}^{\infty}\left(\mathbb{R}^{+} ; \mathbb{R}\right)} t\right] e^{\left(G_{1}+M\right) t} \\
& +4\left[1+\left(G_{1}+M\right) t\right] F_{\infty} t e^{\left(G_{1}+M\right) t} .
\end{aligned}
$$

If $x<\sigma(t)$, then

$$
\begin{aligned}
\mathrm{TV}(u(\cdot, x) ;[0, t]) \leq & {\left[\operatorname{TV}\left(u_{o} ; \mathbb{R}^{+}\right)+\frac{1}{\check{g}} \mathrm{TV}(b(\cdot) ;[0, t])\right] e^{\left(G_{1}+M\right) t} } \\
& +2\left[1+\left(G_{1}+M\right) t\right]\left\|u_{o}\right\|_{\mathbf{L}^{\infty}\left(\mathbb{R}^{+} ; \mathbb{R}\right)} e^{\left(G_{1}+M\right) t} \\
& +\frac{1}{\check{g}}\left[2+3\left(G_{1}+M\right) t+\frac{G_{\infty}}{\check{g}}\right]\|b\|_{\mathbf{L}^{\infty}([0, t] ; \mathbb{R})} e^{\left(G_{1}+M\right) t} \\
& +2\left(7+6\left(G_{1}+M\right) t\right) F_{\infty} t e^{\left(G_{1}+M\right) t} .
\end{aligned}
$$

(SP.5) Fix a positive $W$. For any $w \in\left(\mathbf{C}^{\mathbf{1}} \cap \mathbf{B V}\right)(J ;[-W, W])$,

$$
\begin{aligned}
\mathrm{TV}\left(\int_{\mathbb{R}^{+}} w(\cdot, x) u(\cdot, x) \mathrm{d} x ;[0, t]\right) \leq & \|u\|_{\mathbf{L}^{\infty}\left([0, t] \times \mathbb{R}^{+} ; \mathbb{R}\right)} \int_{\mathbb{R}^{+}} \operatorname{TV}(w(\cdot, x) ;[0, t]) \mathrm{d} x \\
& +W \int_{\mathbb{R}^{+}} \operatorname{TV}(u(\cdot, x) ;[0, t]) \mathrm{d} x
\end{aligned}
$$

(SP.6) For every $t \in J$, there exists a positive $\mathcal{L}$ dependent on $\left\|u_{o}\right\|_{\mathbf{L}^{1}\left(\mathbb{R}^{+} ; \mathbb{R}\right)}$ and on the constants in $(\mathbf{f}),(\boldsymbol{g}),(\boldsymbol{m})$ and $(\mathbf{b})$, such that, for $t^{\prime}, t^{\prime \prime} \in[0, t]$,

$$
\left\|u\left(t^{\prime}\right)-u\left(t^{\prime \prime}\right)\right\|_{\mathbf{L}^{1}\left(\mathbb{R}^{+} ; \mathbb{R}\right)} \leq \mathcal{L}\left|t^{\prime \prime}-t^{\prime}\right| .
$$

(SP.7) If $u_{o} \geq 0, f \geq 0$ and $b \geq 0$, then $u(t) \geq 0$ for all $t$. 
Remark 4.6. The boundedness of the space variation of $f$ required in $(f)$ is necessary. Indeed, consider Problem (4.8) with $g(t, x)=1, m(t, x)=0, f(t, x)=\sin \frac{1}{x-t}, u_{o}(x)=0$ and $b(t)=0$. The solution is $u(t, x)=t \sin \frac{1}{x-t}$ which has unbounded total variation in space for all $t>0$.

Proof of Lemma 4.5. We prove the different items separately.

(SP.1): A standard integration along characteristics is sufficient to prove it.

(SP.2): These bounds are an immediate consequence of (f), (g), (m) and (4.9).

(SP.3); We clearly have

$$
\operatorname{TV}(u(t))=\operatorname{TV}(u(t, \cdot),[0, \sigma(t)[)+|u(t, \sigma(t)+)-u(t, \sigma(t)-)|+\operatorname{TV}(u(t, \cdot),] \sigma(t),+\infty[)
$$

and we estimate the three terms in the right hand side of (4.20) separately. Begin with the first one, using the second expression in (4.9):

$$
\begin{aligned}
& \operatorname{TV}(u(t, \cdot),[0, \sigma(t)[) \\
\leq & \operatorname{TV}\left(\frac{b(\cdot)}{g(\cdot, 0)} ;[0, t]\right) e^{\left(G_{1}+M\right) t}+\left(G_{1}+M\right) t \frac{\|b\|_{\mathbf{L}^{\infty}([0, t] ; \mathbb{R})}}{\check{g}} e^{\left(G_{1}+M\right) t} \\
& +\int_{0}^{t} \operatorname{TV}(f(\tau, X(\tau ; t, \cdot)) \mathcal{E}(\tau, t, \cdot)) \mathrm{d} \tau+\|f\|_{\mathbf{L}^{\infty}\left([0, t] \times \mathbb{R}^{+} ; \mathbb{R}\right)} e^{\left(G_{1}+M\right) t} \mathrm{TV}(T(0 ; t, \cdot)) \\
\leq & \left(\frac{1}{\check{g}} \operatorname{TV}(b ;[0, t])+\frac{1}{\check{g}^{2}} G_{\infty}\|b\|_{\mathbf{L}^{\infty}([0, t] ; \mathbb{R})}+\left(G_{1}+M\right) t \frac{\|b\|_{\mathbf{L}^{\infty}([0, t] ; \mathbb{R})}}{\check{g}}\right) e^{\left(G_{1}+M\right) t} \\
& +\left(G_{1}+M\right) F_{\infty} t^{2} e^{\left(G_{1}+M\right) t}+\sup _{t \in J} \operatorname{TV}(f(t, \cdot)) t e^{\left(G_{1}+M\right) t}+F_{\infty} t e^{\left(G_{1}+M\right) t} \\
\leq & \frac{1}{\check{g}}\left(\operatorname{TV}(b ;[0, t])+\left(\frac{G_{\infty}}{\check{g}}+\left(G_{1}+M\right) t\right)\|b\|_{\mathbf{L}^{\infty}([0, t] ; \mathbb{R})}\right) e^{\left(G_{1}+M\right) t} \\
& +\left(\left(G_{1}+M\right) t+2\right) F_{\infty} t e^{\left(G_{1}+M\right) t} .
\end{aligned}
$$

Concerning the second term in (4.20), the following rough estimate is sufficient for later use:

$$
\begin{aligned}
|u(t, \sigma(t)+)-u(t, \sigma(t)-)| & \leq 2\|u(t)\|_{\mathbf{L}^{\infty}\left(\mathbb{R}^{+} ; \mathbb{R}\right)} \\
& \leq 2\left(\left\|u_{o}\right\|_{\mathbf{L}^{\infty}\left(\mathbb{R}^{+} ; \mathbb{R}\right)}+\frac{1}{\check{g}}\|b\|_{\mathbf{L}^{\infty}([0, t] ; \mathbb{R})}+F_{\infty} t\right) e^{\left(G_{1}+M\right) t} .
\end{aligned}
$$

The latter term in (4.20) reads

$$
\begin{aligned}
\operatorname{TV}(u(t, \cdot),] \sigma(t),+\infty[) \\
\leq \quad\left(G_{1}+M\right)\left\|u_{o}\right\|_{\mathbf{L}^{\infty}\left(\mathbb{R}^{+} ; \mathbb{R}\right)} t e^{\left(G_{1}+M\right) t}+\operatorname{TV}\left(u_{o}\right) e^{\left(G_{1}+M\right) t} \\
\quad+\int_{0}^{t} \operatorname{TV}(f(\tau, X(\tau ; t, \cdot)) \mathcal{E}(\tau, t, \cdot)) \mathrm{d} \tau
\end{aligned}
$$




$$
\begin{aligned}
\leq & \left(\left(G_{1}+M\right)\left\|u_{o}\right\|_{\mathbf{L}^{\infty}\left(\mathbb{R}^{+} ; \mathbb{R}\right)} t+\operatorname{TV}\left(u_{o}\right)\right) e^{\left(G_{1}+M\right) t} \\
& +\left(G_{1}+M\right)\|f\|_{\mathbf{L}^{\infty}\left([0, t] \times \mathbb{R}^{+} ; \mathbb{R}\right)} t^{2} e^{\left(G_{1}+M\right) t}+\sup _{t \in J} \operatorname{TV}(f(t, \cdot)) t e^{\left(G_{1}+M\right) t} \\
& \leq\left(\left(G_{1}+M\right)\left(\left\|u_{o}\right\|_{\mathbf{L}^{\infty}(\mathbb{R}+; \mathbb{R})}+F_{\infty} t\right) t+\operatorname{TV}\left(u_{o}\right)+F_{\infty} t\right) e^{\left(G_{1}+M\right) t} .
\end{aligned}
$$

Using now (4.20),

$$
\begin{aligned}
& \operatorname{TV}\left(u(t, \cdot) ; \mathbb{R}^{+}\right) \\
\leq & {\left[\frac{1}{\check{g}} \mathrm{TV}(b ;[0, t])+\frac{1}{\check{g}}\left(\frac{G_{\infty}}{\check{g}}+\left(G_{1}+M\right) t\right)\|b\|_{\mathbf{L}^{\infty}([0, t] ; \mathbb{R})}\right.} \\
& +\left(\left(G_{1}+M\right) t+2\right) F_{\infty} t+2\left(\left\|u_{o}\right\|_{\mathbf{L}^{\infty}\left(\mathbb{R}^{+} ; \mathbb{R}\right)}+\frac{1}{\check{g}}\|b\|_{\mathbf{L}^{\infty}([0, t] ; \mathbb{R})}+F_{\infty} t\right) \\
& \left.+\left(G_{1}+M\right) t\left(\left\|u_{o}\right\|_{\mathbf{L}^{\infty}\left(\mathbb{R}^{+} ; \mathbb{R}\right)}+F_{\infty} t\right)+\mathrm{TV}\left(u_{o}\right)+F_{\infty} t\right] e^{\left(G_{1}+M\right) t} \\
\leq & {\left[\frac{1}{\check{g}}\left(2+\frac{G_{\infty}}{\check{g}}+\left(G_{1}+M\right) t\right)\|b\|_{\mathbf{L}^{\infty}([0, t] ; \mathbb{R})}+\frac{1}{\check{g}} \mathrm{TV}(b ;[0, t])+\left(\left(G_{1}+M\right) t+5\right) F_{\infty} t\right.} \\
& \left.+\left(2+\left(G_{1}+M\right) t\right)\left\|u_{o}\right\|_{\mathbf{L}^{\infty}\left(\mathbb{R}^{+} ; \mathbb{R}\right)}+\mathrm{TV}\left(u_{o}\right)\right] e^{\left(G_{1}+M\right) t} \\
\leq & \mathcal{H}(t)\left(F_{\infty} t+\frac{1}{\check{g}}\|b\|_{\mathbf{L}^{\infty}([0, t] ; \mathbb{R})}+\frac{1}{\check{g}} \mathrm{TV}(b ;[0, t])+\left\|u_{o}\right\|_{\mathbf{L}^{\infty}\left(\mathbb{R}^{+} ; \mathbb{R}\right)}+\mathrm{TV}\left(u_{o}\right)\right)
\end{aligned}
$$

we prove (4.16).

(SP.4): First, in view of an application of Lemma 4.1, compute

$$
\begin{array}{rlr} 
& \operatorname{TV}(f(\tau, X(\tau ; \cdot, x)) \mathcal{E}(\tau, \cdot, x) ;[0, t]) & \text { [Use (4.2)] } \\
\leq & \operatorname{TV}(f(\tau, X(\tau ; \cdot, x)) ;[0, t])\|\mathcal{E}(\tau, \cdot, x)\|_{\mathbf{L}^{\infty}([0, t] ; \mathbb{R})} & \text { [Use (4.3) and (4.12)] }] \\
& +\|f(\tau, X(\tau ; \cdot, x))\|_{\mathbf{L}^{\infty}([0, t] ; \mathbb{R})} \operatorname{TV}(\mathcal{E}(\tau, \cdot, x) ;[0, t]) & \text { [Use (4.14)] } \\
\leq & e^{\left(G_{1}+M\right) t} \operatorname{TV}\left(f(\tau, \cdot) ; \mathbb{R}^{+}\right) & \\
& +2\left(G_{1}+M\right) t e^{\left(G_{1}+M\right) t}\|f\|_{\mathbf{L}^{\infty}([0, t] \times \mathbb{R} ; \mathbb{R})} . &
\end{array}
$$

Thus, using (f), we deduce that

$$
\operatorname{TV}(f(\tau, X(\tau ; \cdot, x)) \mathcal{E}(\tau, \cdot, x) ;[0, t]) \leq\left(1+2\left(G_{1}+M\right) t\right) F_{\infty} e^{\left(G_{1}+M\right) t} .
$$

First consider the simple case $x>\sigma(t)$; we have

$$
\begin{aligned}
& \operatorname{TV}(u(\cdot, x) ;[0, t]) \\
\leq & \operatorname{TV}\left(u_{o}(X(0 ; \cdot, x)) \mathcal{E}(0, \cdot, x) ;[0, t]\right) \\
& +\operatorname{TV}\left(\int_{0}^{\cdot} f(\tau, X(\tau ; \cdot, x)) \mathcal{E}(\tau, \cdot, x) \mathrm{d} \tau ;[0, t]\right)
\end{aligned}
$$




$$
\begin{aligned}
\leq & \operatorname{TV}\left(u_{o}(X(0 ; \cdot, x)) ;[0, t]\right)\|\mathcal{E}(0, \cdot, x)\|_{\mathbf{L}^{\infty}\left([0, t] ; \mathbb{R}^{+}\right)} \\
& +\left\|u_{o}(X(0 ; \cdot, x))\right\|_{\mathbf{L}^{\infty}\left([0, t] ; \mathbb{R}^{+}\right)} \operatorname{TV}(\mathcal{E}(0, \cdot, x) ;[0, t]) \\
& +2 \sup _{\tau \in[0, t]} \operatorname{TV}(f(\tau, X(\tau ; \cdot, x)) \mathcal{E}(\tau, \cdot, x) ;[0, t]) t \\
& +2\|f\|_{\mathbf{L}^{\infty}\left([0, t] \times \mathbb{R}^{+} ; \mathbb{R}\right)}\|\mathcal{E}\|_{\mathbf{L}^{\infty}\left([0, t]^{2} \times \mathbb{R}^{+} ; \mathbb{R}\right)} t \\
\leq & \operatorname{TV}\left(u_{o} ; \mathbb{R}^{+}\right) e^{\left(G_{1}+M\right) t}+2\left(G_{1}+M\right)\left\|u_{o}\right\|_{\mathbf{L}^{\infty}\left(\mathbb{R}^{+} ; \mathbb{R}\right)} t e^{\left(G_{1}+M\right) t} \\
& +2\left(1+2\left(G_{1}+M\right) t\right) F_{\infty} t e^{\left(G_{1}+M\right) t}+2 F_{\infty} t e^{\left(G_{1}+M\right) t} .
\end{aligned}
$$

[Use (4.12)]

[Use (4.14)]

[Use (4.21)]

[Use (f) and (4.12)]

Now consider the case $x<\sigma(t)$, i.e. $\Sigma(x)<t$. We clearly have

$$
\operatorname{TV}(u(\cdot, x) ;[0, t]) \leq \operatorname{TV}(u(\cdot, x) ;[0, \Sigma(x)])+\operatorname{TV}(u(\cdot, x) ;[\Sigma(x), t])+2\|u\|_{\mathbf{L}^{\infty}\left([0, t] \times \mathbb{R}^{+} ; \mathbb{R}\right)} \cdot
$$

Let us estimate the second term $\operatorname{TV}(u(\cdot, x) ;[\Sigma(x), t])$.

$$
\begin{aligned}
& \operatorname{TV}(u(\cdot, x) ;[\Sigma(x), t]) \\
\leq & \operatorname{TV}\left(\frac{b(T(0 ; \cdot, x))}{g(T(0 ; \cdot, x), 0)} \mathcal{E}(T(0 ; \cdot, x), \cdot, x) ;[\Sigma(x), t]\right) \\
& +\mathrm{TV}\left(\int_{T(0 ; \cdot, x)} f(\tau, X(\tau ; \cdot, x)) \mathcal{E}(\tau, \cdot, x) \mathrm{d} \tau ;[\Sigma(x), t]\right) \\
\leq & \frac{1}{\check{g}} \operatorname{TV}(b(T(0 ; \cdot, x)) ;[\Sigma(x), t])\|\mathcal{E}(T(0 ; \cdot, x), \cdot, x)\|_{\mathbf{L}^{\infty}([0, t] ; \mathbb{R})} \\
& +\frac{1}{\check{g}^{2}} \operatorname{TV}(g(T(0 ; \cdot, x), 0) ;[\Sigma(x), t])\|b(T(0 ; \cdot, x)) \mathcal{E}(T(0 ; \cdot, x), \cdot, x)\|_{\mathbf{L}^{\infty}([0, t] ; \mathbb{R})} \\
& +\frac{1}{\check{g}}\|b(T(0 ; \cdot, x))\|_{\mathbf{L}^{\infty}([0, t] ; \mathbb{R})} \operatorname{TV}(\mathcal{E}(T(0 ; \cdot, x), \cdot, x) ;[\Sigma(x), t]) \\
& +4(t-\Sigma(x)) \sup _{\tau \in[\Sigma(x), t]} \operatorname{TV}(f(\tau, X(\tau ; \cdot, x)) \mathcal{E}(\tau, \cdot, x) ;[\Sigma(x), t]) \\
& +4(t-\Sigma(x))\|f(\cdot, X(\cdot ; \cdot, x)) \mathcal{E}(\cdot, \cdot, x)\|_{\mathbf{L}^{\infty}([\Sigma(x), t] ; \mathbb{R})} \\
\leq & \frac{1}{\check{g}}\left[\operatorname{TV}(b(\cdot) ;[0, t])+\left(3\left(G_{1}+M\right) t+\frac{G_{\infty}}{\check{g}}\right)\|b\|_{\mathbf{L}^{\infty}([0, t] ; \mathbb{R})}\right] e^{\left(G_{1}+M\right) t} \\
& +4(t-\Sigma(x))\left[2+2 t\left(G_{1}+M\right)\right] F_{\infty} e^{\left(G_{1}+M\right) t} .
\end{aligned}
$$

[Use (4.9)]

Therefore, using (SP.2), we deduce that

$$
\begin{aligned}
& \operatorname{TV}(u(\cdot, x) ;[0, t]) \\
\leq & {\left[\operatorname{TV}\left(u_{o} ; \mathbb{R}^{+}\right)+\frac{1}{\check{g}} \operatorname{TV}(b(\cdot) ;[0, t])\right] e^{\left(G_{1}+M\right) t}+2\left[1+\left(G_{1}+M\right) t\right]\left\|u_{o}\right\|_{\mathbf{L}^{\infty}(\mathbb{R}+; \mathbb{R})} e^{\left(G_{1}+M\right) t} } \\
& +\frac{1}{\check{g}}\left[2+3\left(G_{1}+M\right) t+\frac{G_{\infty}}{\check{g}}\right]\|b\|_{\mathbf{L}^{\infty}([0, t] ; \mathbb{R})} e^{\left(G_{1}+M\right) t}+2\left(7+6\left(G_{1}+M\right) t\right) F_{\infty} t e^{\left(G_{1}+M\right) t} .
\end{aligned}
$$

This completes the proof of (SP.4). 
(SP.5): Using the already obtained estimates, we have:

$$
\begin{aligned}
& \operatorname{TV}\left(\int_{J} w(\cdot, x) u(\cdot, x) \mathrm{d} x ;[0, t]\right) \\
& \leq \int_{J} \operatorname{TV}(w(\cdot, x) u(\cdot, x) ;[0, t]) \mathrm{d} x \\
& \leq \int_{J}\left(\operatorname{TV}(w(\cdot, x) ;[0, t])\|u(\cdot, x)\|_{\mathbf{L}^{\infty}([0, t] ; \mathbb{R})}+\|w(\cdot, x)\|_{\mathbf{L}^{\infty}([0, t] ; \mathbb{R})} \operatorname{TV}(u(\cdot, x) ;[0, t])\right) \mathrm{d} x \quad[\text { By (4.2) }] \\
& \leq \int_{J} \operatorname{TV}(w(\cdot, x) ;[0, t]) \mathrm{d} x\|u\|_{\mathbf{L}^{\infty}([0, t] \times J ; \mathbb{R})}+W \int_{J} \operatorname{TV}(u(\cdot, x) ;[0, t]) \mathrm{d} x
\end{aligned}
$$

(SP.6): Fix $t^{\prime}, t^{\prime \prime} \in J$ with $t^{\prime}<t^{\prime \prime}$. Then,

$$
\left\|u\left(t^{\prime \prime}\right)-u\left(t^{\prime}\right)\right\|_{\mathbf{L}^{1}\left(\mathbb{R}^{+} ; \mathbb{R}\right)}=\int_{0}^{X\left(t^{\prime \prime} ; t^{\prime}, 0\right)}\left|u\left(t^{\prime \prime}, x\right)-u\left(t^{\prime}, x\right)\right| \mathrm{d} x+\int_{X\left(t^{\prime \prime} ; t^{\prime}, 0\right)}^{+\infty}\left|u\left(t^{\prime \prime}, x\right)-u\left(t^{\prime}, x\right)\right| \mathrm{d} x
$$

We estimate the two latter terms above separately:

$$
\begin{aligned}
& \int_{0}^{X\left(t^{\prime \prime} ; t^{\prime}, 0\right)}\left|u\left(t^{\prime \prime}, x\right)-u\left(t^{\prime}, x\right)\right| \mathrm{d} x \\
\leq & \left(\left\|u\left(t^{\prime \prime}\right)\right\|_{\mathbf{L}^{\infty}\left(\mathbb{R}^{+} ; \mathbb{R}\right)}+\left\|u\left(t^{\prime}\right)\right\|_{\mathbf{L}^{\infty}\left(\mathbb{R}^{+} ; \mathbb{R}\right)}\right) X\left(t^{\prime \prime} ; t^{\prime}, 0\right) \\
\leq & 2 \hat{g}\left(\left\|u_{o}\right\|_{\mathbf{L}^{\infty}\left(\mathbb{R}^{+} ; \mathbb{R}\right)}+\frac{1}{\grave{g}}\|b\|_{\mathbf{L}^{\infty}\left(\left[0, t^{\prime \prime}\right] ; \mathbb{R}\right)}+\|f\|_{\mathbf{L}^{\infty}\left(\left[0, t^{\prime \prime}\right] \times \mathbb{R}^{+} ; \mathbb{R}\right)} t^{\prime \prime}\right)\left(t^{\prime \prime}-t^{\prime}\right) e^{\left(G_{1}+M\right) t^{\prime \prime}} .
\end{aligned}
$$

Passing to the next term,

$$
\begin{aligned}
& \int_{X\left(t^{\prime \prime} ; t^{\prime}, 0\right)}^{+\infty}\left|u\left(t^{\prime \prime}, x\right)-u\left(t^{\prime}, x\right)\right| \mathrm{d} x \\
\leq & \int_{X\left(t^{\prime \prime} ; t^{\prime}, 0\right)}^{+\infty}\left|u\left(t^{\prime}, X\left(t^{\prime} ; t^{\prime \prime}, x\right)\right)\right|\left|\mathcal{E}\left(t^{\prime}, t^{\prime \prime}, x\right)-1\right| \mathrm{d} x \\
& +\int_{X\left(t^{\prime \prime} ; t^{\prime}, 0\right)}^{+\infty}\left|u\left(t^{\prime}, X\left(t^{\prime} ; t^{\prime \prime}, x\right)\right)-u\left(t^{\prime}, x\right)\right| \mathrm{d} x \\
& +\int_{X\left(t^{\prime \prime} ; t^{\prime}, 0\right)}^{+\infty} \int_{t^{\prime}}^{t^{\prime \prime}}\left|f\left(\tau, X\left(\tau ; t^{\prime \prime}, x\right)\right)\right| \mathcal{E}\left(\tau, t^{\prime \prime}, x\right) \mathrm{d} \tau \mathrm{d} x \\
\leq & \left\|u\left(t^{\prime}\right)\right\|_{\mathbf{L}^{1}\left(\mathbb{R}^{+} ; \mathbb{R}\right)}\left(e^{\left(G_{1}+M\right)\left(t^{\prime \prime}-t^{\prime}\right)}-1\right) \\
& +\mathrm{TV}\left(u\left(t^{\prime}\right)\right)\left\|X\left(t^{\prime} ; t^{\prime \prime}, \cdot\right)-\cdot\right\|_{\mathbf{L}^{\infty}\left(\mathbb{R}^{+} ; \mathbb{R}\right)} \\
& +\sup _{\left[t^{\prime}, t^{\prime \prime}\right]}\|f(t)\|_{\mathbf{L}^{1}\left(\mathbb{R}^{+} ; \mathbb{R}\right)} e^{\left(G_{1}+M\right)\left(t^{\prime \prime}-t^{\prime}\right)\left(t^{\prime \prime}-t^{\prime}\right)} \\
\leq & \mathcal{L}\left(t^{\prime \prime}-t^{\prime}\right),
\end{aligned}
$$

completing the proof of (4.19).

(SP.7); This bound is a direct consequence of (4.9). 
Lemma 4.7. Let (g) holds. Fix $u_{o}^{\prime}, u_{o}^{\prime \prime} \in\left(\mathbf{L}^{1} \cap \mathbf{B V}\right)\left(\mathbb{R}^{+} ; \mathbb{R}\right), b^{\prime}, b^{\prime \prime}$ satisfying $(\mathbf{b})$, $m^{\prime}, m^{\prime \prime}$ satisfying $(\mathbf{m})$, and $f^{\prime}, f^{\prime \prime}$ satisfying (f). Call $u^{\prime}$ and $u^{\prime \prime}$ the solutions to

$$
\left\{\begin{array} { l } 
{ \partial _ { t } u + \partial _ { x } ( g ( t , x ) u ) + m ^ { \prime } ( t , x ) u = f ^ { \prime } ( t , x ) } \\
{ u ( 0 , x ) = u _ { o } ^ { \prime } ( x ) } \\
{ g ( t , 0 ) u ( t , 0 + ) = b ^ { \prime } ( t ) }
\end{array} \text { and } \left\{\begin{array}{l}
\partial_{t} u+\partial_{x}(g(t, x) u)+m^{\prime \prime}(t, x) u=f^{\prime \prime}(t, x) \\
u(0, x)=u_{o}^{\prime \prime}(x) \\
g(t, 0) u(t, 0+)=b^{\prime \prime}(t)
\end{array}\right.\right.
$$

Then,

(SP.8) The following stability conditions hold:

$$
\begin{aligned}
& \quad\left\|u^{\prime}(t)-u^{\prime \prime}(t)\right\|_{\mathbf{L}^{1}\left(\mathbb{R}^{+} ; \mathbb{R}\right)} \\
& \leq e^{M t}\left\|u_{o}^{\prime}-u_{o}^{\prime \prime}\right\|_{\mathbf{L}^{\mathbf{1}}\left(\mathbb{R}^{+} ; \mathbb{R}\right)}+e^{2\left(G_{1}+M\right) t}\left(2\left\|f^{\prime}-f^{\prime \prime}\right\|_{\mathbf{L}^{\mathbf{1}}\left(J \times \mathbb{R}^{+} ; \mathbb{R}\right)}+\left\|b^{\prime}-b^{\prime \prime}\right\|_{\mathbf{L}^{\mathbf{1}}([0, t] ; \mathbb{R})}\right) \\
& \quad+e^{\left(2 G_{1}+M\right) t}\left[\left\|u_{o}^{\prime \prime}\right\|_{\mathbf{L}^{\mathbf{1}\left(\mathbb{R}^{+} ; \mathbb{R}\right)}}+2 t F_{1}+\left\|b^{\prime \prime}\right\|_{\mathbf{L}^{\mathbf{1}}([0, t] ; \mathbb{R})}\right] t\left\|m^{\prime}-m^{\prime \prime}\right\|_{\mathbf{L}^{\infty}\left([0, t] \times \mathbb{R}^{+} ; \mathbb{R}\right)}
\end{aligned}
$$

and

$$
\begin{aligned}
& \left\|u^{\prime}(t)-u^{\prime \prime}(t)\right\|_{\mathbf{L}^{1}\left(\mathbb{R}^{+} ; \mathbb{R}\right)} \\
& \leq e^{M t}\left\|u_{o}^{\prime}-u_{o}^{\prime \prime}\right\|_{\mathbf{L}^{1}\left(\mathbb{R}^{+} ; \mathbb{R}\right)}+e^{2\left(G_{1}+M\right) t}\left(2\left\|f^{\prime}-f^{\prime \prime}\right\|_{\mathbf{L}^{1}\left(J \times \mathbb{R}^{+} ; \mathbb{R}\right)}+\left\|b^{\prime}-b^{\prime \prime}\right\|_{\mathbf{L}^{1}([0, t] ; \mathbb{R})}\right) \\
& \quad+e^{\left(2 G_{1}+M\right) t}\left[\left\|u_{o}^{\prime \prime}\right\|_{\mathbf{L}^{\infty}\left(\mathbb{R}^{+} ; \mathbb{R}\right)}+2 t F_{\infty}+\frac{\left\|b^{\prime \prime}\right\|_{\mathbf{L}^{\infty}([0, t] ; \mathbb{R})}}{\check{g}}\right]\left\|m^{\prime}-m^{\prime \prime}\right\|_{\mathbf{L}^{1}\left([0, t] \times \mathbb{R}^{+} ; \mathbb{R}\right)} .
\end{aligned}
$$

(SP.9) The following monotonicity property holds:

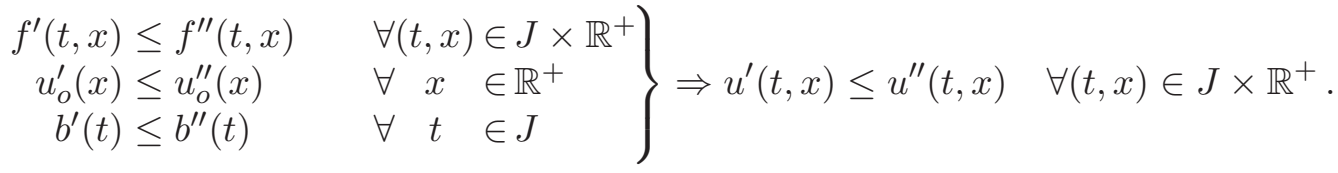

(SP.10) If $\bar{x}>0$ and $\sigma(t)<\bar{x}$, then

$$
\begin{aligned}
& \left\|u^{\prime}(\cdot, \bar{x})-u^{\prime \prime}(\cdot, \bar{x})\right\|_{\mathbf{L}^{1}([0, t] ; \mathbb{R})} \\
& \leq e^{\left(G_{1}+M\right) t} t\left[e^{G_{1} t}\left\|u_{o}^{\prime}\right\|_{\mathbf{L}^{1}(\mathbb{R}+; \mathbb{R})}+t^{2} F_{\infty}\right]\left\|m^{\prime}-m^{\prime \prime}\right\|_{\mathbf{L}^{\infty}\left([0, t] \times \mathbb{R}^{+} ; \mathbb{R}\right)} \\
& +e^{M t}\left\|u_{o}^{\prime}-u_{o}^{\prime \prime}\right\|_{\mathbf{L}^{1}\left(\mathbb{R}^{+} ; \mathbb{R}\right)}+e^{\left(2 G_{1}+M\right) t}\left\|f^{\prime}-f^{\prime \prime}\right\|_{\mathbf{L}^{1}\left([0, t] \times \mathbb{R}^{+} ; \mathbb{R}\right)}
\end{aligned}
$$

and

$$
\begin{aligned}
& \left\|u^{\prime}(\cdot, \bar{x})-u^{\prime \prime}(\cdot, \bar{x})\right\|_{\mathbf{L}^{1}([0, t] ; \mathbb{R})} \\
\leq & \frac{e^{\left(G_{1}+M\right) t}}{G_{\infty}}\left[e^{G_{1} t}\left\|u_{o}^{\prime}\right\|_{\mathbf{L}^{\infty}\left(\mathbb{R}^{+} ; \mathbb{R}\right)}+t F_{\infty}\right]\left\|m^{\prime}-m^{\prime \prime}\right\|_{\mathbf{L}^{1}\left([0, t] \times \mathbb{R}^{+} ; \mathbb{R}\right)} \\
& +e^{M t}\left\|u_{o}^{\prime}-u_{o}^{\prime \prime}\right\|_{\mathbf{L}^{1}\left(\mathbb{R}^{+} ; \mathbb{R}\right)}+e^{\left(2 G_{1}+M\right) t}\left\|f^{\prime}-f^{\prime \prime}\right\|_{\mathbf{L}^{1}\left([0, t] \times \mathbb{R}^{+} ; \mathbb{R}\right)}
\end{aligned}
$$


Proof. The stability bounds (4.24) and (4.25) can be easily proved using the explicit formula (4.9) and the estimates of Lemma 4.4. Also the monotonicity property (4.26) directly follows from (4.9).

We pass to the proof of the estimate (4.27). The proof of the estimate (4.28) is completely analogous. Using (4.9) with the condition $\sigma(t)<\bar{x}$ we deduce that

$$
\begin{aligned}
\left\|u^{\prime}(\cdot, \bar{x})-u^{\prime \prime}(\cdot, \bar{x})\right\|_{\mathbf{L}^{1}([0, t] ; \mathbb{R}) \leq} & \int_{0}^{t}\left|u_{o}^{\prime}(X(0 ; s, \bar{x}))\right|\left|\mathcal{E}^{\prime}(0, s, \bar{x})-\mathcal{E}^{\prime \prime}(0, s, \bar{x})\right| \mathrm{d} s \\
& +\int_{0}^{t}\left|u_{o}^{\prime}(X(0 ; s, \bar{x}))-u_{o}^{\prime \prime}(X(0 ; s, \bar{x}))\right|\left|\mathcal{E}^{\prime \prime}(0, s, \bar{x})\right| \mathrm{d} s \\
& +\int_{0}^{t} \int_{0}^{s}\left|f^{\prime}(\tau, X(\tau ; s, \bar{x}))\right|\left|\mathcal{E}^{\prime}(\tau, s, \bar{x})-\mathcal{E}^{\prime \prime}(\tau, s, \bar{x})\right| \mathrm{d} \tau \mathrm{d} s \\
& +\int_{0}^{t} \int_{0}^{s}\left|f^{\prime}(\tau, X(\tau ; s, \bar{x}))-f^{\prime \prime}(\tau, X(\tau ; s, \bar{x}))\right|\left|\mathcal{E}^{\prime \prime}(\tau, s, \bar{x})\right| \mathrm{d} \tau \mathrm{d} s .
\end{aligned}
$$

We now estimate all the terms in the previous inequality. Using (4.10), (m) and (g) we have

$$
\begin{aligned}
& \int_{0}^{t}\left|u_{o}^{\prime}(X(0 ; s, \bar{x}))\right|\left|\mathcal{E}^{\prime}(0, s, \bar{x})-\mathcal{E}^{\prime \prime}(0, s, \bar{x})\right| \mathrm{d} s \\
\leq & e^{\left(G_{1}+M\right) t} t\left\|m^{\prime}-m^{\prime \prime}\right\|_{\mathbf{L}^{\infty}\left([0, t] \times \mathbb{R}^{+} ; \mathbb{R}\right)} \int_{0}^{t}\left|u_{o}^{\prime}(X(0 ; s, \bar{x}))\right| \mathrm{d} s \\
\leq & e^{\left(2 G_{1}+M\right) t} t\left\|m^{\prime}-m^{\prime \prime}\right\|_{\mathbf{L}^{\infty}\left([0, t] \times \mathbb{R}^{+} ; \mathbb{R}\right)}\left\|u_{o}^{\prime}\right\|_{\mathbf{L}^{1}\left(\mathbb{R}^{+} ; \mathbb{R}\right)} .
\end{aligned}
$$

Using (4.10), and (m), we deduce that

$$
\int_{0}^{t}\left|u_{o}^{\prime}(X(0 ; s, \bar{x}))-u_{o}^{\prime \prime}(X(0 ; s, \bar{x}))\right|\left|\mathcal{E}^{\prime \prime}(0, s, \bar{x})\right| \mathrm{d} s \leq e^{M t}\left\|u_{o}^{\prime}-u_{o}^{\prime \prime}\right\|_{\mathbf{L}^{1}\left(\mathbb{R}^{+} ; \mathbb{R}\right)} .
$$

Using (4.10), (m), (g) and (f) we have

$$
\begin{aligned}
& \int_{0}^{t} \int_{0}^{s}\left|f^{\prime}(\tau, X(\tau ; s, \bar{x}))\right|\left|\mathcal{E}^{\prime}(\tau, s, \bar{x})-\mathcal{E}^{\prime \prime}(\tau, s, \bar{x})\right| \mathrm{d} \tau \mathrm{d} s \\
\leq & e^{\left(G_{1}+M\right) t} t\left\|m^{\prime}-m^{\prime \prime}\right\|_{\mathbf{L}^{\infty}\left([0, t] \times \mathbb{R}^{+} ; \mathbb{R}\right)} \int_{0}^{t} \int_{0}^{s}\left|f^{\prime}(\tau, X(\tau ; s, \bar{x}))\right| \mathrm{d} \tau \mathrm{d} s \\
\leq & e^{\left(G_{1}+M\right) t} t^{3}\left\|m^{\prime}-m^{\prime \prime}\right\|_{\mathbf{L}^{\infty}\left([0, t] \times \mathbb{R}^{+} ; \mathbb{R}\right)} F_{\infty} .
\end{aligned}
$$

Finally using (4.12) and (g) we get

$$
\begin{aligned}
& \int_{0}^{t} \int_{0}^{s}\left|f^{\prime}(\tau, X(\tau ; s, \bar{x}))-f^{\prime \prime}(\tau, X(\tau ; s, \bar{x}))\right|\left|\mathcal{E}^{\prime \prime}(\tau, s, \bar{x})\right| \mathrm{d} \tau \mathrm{d} s \\
\leq & e^{\left(G_{1}+M\right) t} \int_{0}^{t} \int_{0}^{s}\left|f^{\prime}(\tau, X(\tau ; s, \bar{x}))-f^{\prime \prime}(\tau, X(\tau ; s, \bar{x}))\right| \mathrm{d} \tau \mathrm{d} s \\
\leq & e^{\left(G_{1}+M\right) t} e^{G_{1} t} \int_{0}^{t} \int_{\mathbb{R}^{+}}\left|f^{\prime}(\tau, x)-f^{\prime \prime}(\tau, x)\right| \mathrm{d} x \mathrm{~d} \tau \\
\leq & e^{\left(2 G_{1}+M\right) t}\left\|f^{\prime}-f^{\prime \prime}\right\|_{\mathbf{L}^{1}\left([0, t] \times \mathbb{R}^{+} ; \mathbb{R}\right)} .
\end{aligned}
$$


Therefore

$$
\begin{aligned}
\left\|u^{\prime}(\cdot, \bar{x})-u^{\prime \prime}(\cdot, \bar{x})\right\|_{\mathbf{L}^{\mathbf{1}}([0, t] ; \mathbb{R})} \leq & e^{\left(2 G_{1}+M\right) t} t\left\|u_{o}^{\prime}\right\|_{\mathbf{L}^{\mathbf{1}}\left(\mathbb{R}^{+} ; \mathbb{R}\right)}\left\|m^{\prime}-m^{\prime \prime}\right\|_{\mathbf{L}^{\infty}\left([0, t] \times \mathbb{R}^{+} ; \mathbb{R}\right)} \\
& +e^{M t}\left\|u_{o}^{\prime}-u_{o}^{\prime \prime}\right\|_{\mathbf{L}^{1}\left(\mathbb{R}^{+} ; \mathbb{R}\right)} \\
& +e^{\left(G_{1}+M\right) t} t^{3} F_{\infty}\left\|m^{\prime}-m^{\prime \prime}\right\|_{\mathbf{L}^{\infty}\left([0, t] \times \mathbb{R}^{+} ; \mathbb{R}\right)} \\
& +e^{\left(2 G_{1}+M\right) t}\left\|f^{\prime}-f^{\prime \prime}\right\|_{\mathbf{L}^{1}\left([0, t] \times \mathbb{R}^{+} ; \mathbb{R}\right)} \\
\leq & e^{\left(G_{1}+M\right) t} t\left[e^{G_{1} t}\left\|u_{o}^{\prime}\right\|_{\mathbf{L}^{1}\left(\mathbb{R}^{+} ; \mathbb{R}\right)}+t^{2} F_{\infty}\right]\left\|m^{\prime}-m^{\prime \prime}\right\|_{\mathbf{L}^{\infty}\left([0, t] \times \mathbb{R}^{+} ; \mathbb{R}\right)} \\
& +e^{M t}\left\|u_{o}^{\prime}-u_{o}^{\prime \prime}\right\|_{\mathbf{L}^{1}\left(\mathbb{R}^{+} ; \mathbb{R}\right)}+e^{\left(2 G_{1}+M\right) t}\left\|f^{\prime}-f^{\prime \prime}\right\|_{\mathbf{L}^{1}\left([0, t] \times \mathbb{R}^{+} ; \mathbb{R}\right)}
\end{aligned}
$$

concluding the proof of (4.27).

\subsection{Proofs Related to Section 3 - About the IBVP (3.1)}

Proof of Theorem 3.1, Fix $t_{*}>0$, with $t_{*} \in \mathbb{I}$, and let $J=\left[0, t_{*}\right]$. Define the constants

$$
\begin{aligned}
& K_{1}>\left\|u_{o}\right\|_{\mathbf{L}^{1}\left(\mathbb{R}^{+} ; \mathbb{R}^{n}\right)}+B_{1} \\
& K_{\infty}>\max \left\{\begin{array}{l}
\left(1+\frac{n B_{L}}{\check{g}}\right)\left\|u_{o}\right\|_{\mathbf{L}^{\infty}\left(\mathbb{R} ; \mathbb{R}^{n}\right)}+\frac{B_{\infty}}{\check{g}} \\
\left(5+\frac{G_{\infty}}{\check{g}}\right)\left(\frac{2 B_{\infty}}{\check{g}}+\left(1+\frac{n B_{L}}{\check{g}}\right)\left(\left\|u_{o}\right\|_{\mathbf{L}^{\infty}\left(\mathbb{R}^{+} ; \mathbb{R}^{n}\right)}+\operatorname{TV}\left(u_{o} ; \mathbb{R}^{+}\right)\right)\right)
\end{array}\right.
\end{aligned}
$$

and the complete metric space $\left(\mathcal{X}^{n}, d_{\mathcal{X}^{n}}\right)$ where

$$
\begin{aligned}
& \mathcal{X}=\left\{u \in \mathbf{C}^{\mathbf{0}}\left(J ; \mathbf{L}^{\mathbf{1}}\left(\mathbb{R}^{+} ; \mathbb{R}\right)\right): \begin{array}{rl}
\|u\|_{\mathbf{C}^{\mathbf{0}}\left(J ; \mathbf{L}^{\mathbf{1}}\left(\mathbb{R}^{+} ; \mathbb{R}\right)\right)} & \leq K_{1} \\
\sup _{t \in J} \operatorname{TV}\left(u(t, \cdot) ; \mathbb{R}^{\mathbf{0}}\left(J ; \mathbf{L}^{\infty}\left(\mathbb{R}^{+}\right)\right.\right. & \leq K_{\infty} \\
& \leq K_{\infty}
\end{array}\right\} \\
& d_{\mathcal{X}^{n}}\left(u^{\prime}, u^{\prime \prime}\right)=\max _{i \in\{1, \ldots, n\}}\left\|u_{i}^{\prime \prime}-u_{i}^{\prime}\right\|_{\mathbf{C}^{\mathbf{0}}\left(J ; \mathbf{L}^{\mathbf{1}}\left(\mathbb{R}^{+} ; \mathbb{R}\right)\right)} .
\end{aligned}
$$

Define the map $\mathcal{T}: \mathcal{X}^{n} \rightarrow \mathcal{X}^{n}$, such that, for $w=\left(w_{1}, \cdots, w_{n}\right) \in \mathcal{X}^{n}, \mathcal{T}(w)=u$, where $u=\left(u_{1}, \cdots, u_{n}\right)$ solves

$$
\left\{\begin{array}{l}
\partial_{t} u_{i}+\partial_{x}\left(g_{i}(t, x) u_{i}\right)+m_{i}(t, x) u_{i}=f_{i}(t, x) \\
u_{i}(0, x)=u_{i}^{o}(x) \\
u_{i}(t, 0+)=b_{i}(t)
\end{array} \quad i=1, \ldots, n\right.
$$

where

$$
\begin{aligned}
m_{i}(t, x) & =-\left(\alpha_{i}[w(t)](x)\right)_{i}-\left(\gamma_{i}(t, x)\right)_{i} \\
f_{i}(t, x) & =\sum_{j \neq i}\left(\left(\alpha_{i}[w(t)](x)\right)_{j}+\left(\gamma_{i}(t, x)\right)_{j}\right) w_{j}(t, x) \\
b_{i}(t) & =\beta_{i}\left(t, u_{1}\left(t, \bar{x}_{1}-\right), \ldots, u_{n}\left(t, \bar{x}_{n}-\right)\right) .
\end{aligned}
$$

Remark that in the last line above an essential role is going to be played by the assumption $\partial_{u_{j}} \beta_{i}(t, u)=0$ for all $j \geq i$. 
The map $\mathcal{T}$ is well defined. (i.e. $\mathcal{T}(w) \in \mathcal{X}^{n}$ for every $\left.w \in \mathcal{X}^{n}\right)$. Aiming at the use of Lemma 4.5, we verify that the assumptions (g), (m), (f), and (b) therein hold.

(g) holds. It is immediate by (IBVP.1)

(m) holds. The continuity of $x \rightarrow m(t, x)$ follows from (IBVP.2) and (IBVP.3). Observe that the map $t \rightarrow \alpha_{i}[w(t)](x)$ is continuous, indeed:

$$
\begin{aligned}
\left\|\alpha_{i}\left[w\left(t_{2}\right)\right](x)-\alpha_{i}\left[w\left(t_{1}\right)\right](x)\right\| & \leq\left\|\alpha_{i}\left[w\left(t_{2}\right)\right]-\alpha_{i}\left[w\left(t_{1}\right)\right]\right\|_{\mathbf{C}^{0}\left(\mathbb{R}^{+} ; \mathbb{R}^{n}\right)} \\
& \leq\left\|\alpha_{i}\left[w\left(t_{2}\right)-w\left(t_{1}\right)\right]\right\|_{\mathbf{C}^{\mathbf{0}}\left(\mathbb{R}^{+} ; \mathbb{R}^{n}\right)} \\
& \leq A_{L}\left\|w\left(t_{2}\right)-w\left(t_{1}\right)\right\|_{\mathbf{L}^{\mathbf{1}\left(\mathbb{R}^{+} ; \mathbb{R}\right)}}
\end{aligned}
$$

[By (3.4)]

and the fact that $w \in \mathbf{C}^{\mathbf{0}}\left(J ; \mathbf{L}^{\mathbf{1}}\left(\mathbb{R}^{+} ; \mathbb{R}\right)\right)$ allows to conclude.

Fix $\left(t_{1}, x_{1}\right),\left(t_{2}, x_{2}\right)$ in $J \times \mathbb{R}^{+}$and compute, for $i=1, \ldots, n$,

$$
\begin{aligned}
& \left|m_{i}\left(t, x_{2}\right)-m_{i}\left(t, x_{1}\right)\right| \\
\leq & \left|\left(\alpha_{i}[w(t)]\right)_{i}\left(x_{2}\right)-\left(\alpha_{i}[w(t)]\right)_{i}\left(x_{2}\right)\right|+\left|\left(\gamma_{i}\left(t, x_{2}\right)\right)_{i}-\left(\gamma_{i}\left(t, x_{1}\right)\right)_{i}\right| \\
\leq & \left\|\alpha_{i}[w(t)]\left(x_{2}\right)-\alpha_{i}[w(t)]\left(x_{2}\right)\right\|+\left\|\gamma_{i}\left(t, x_{2}\right)-\gamma_{i}\left(t, x_{1}\right)\right\| \\
\leq & A_{2}\left|x_{2}-x_{1}\right|+C_{L}\left|x_{2}-x_{1}\right| .
\end{aligned}
$$

[By (3.6) and (3.7)

Moreover,

$$
\begin{aligned}
\left\|m_{i}\right\|_{\mathbf{L}^{\infty}\left(J \times \mathbb{R}^{+} ; \mathbb{R}\right)} & \leq \operatorname{ess}_{t \in J}\left\|\left(\alpha_{i}[w(t)]\right)_{i}\right\|_{\mathbf{C}^{\mathbf{0}\left(\mathbb{R}^{+} ; \mathbb{R}\right)}}+\left\|\gamma_{i}\right\|_{\mathbf{L}^{\infty}\left(J \times \mathbb{R}^{+} ; \mathbb{R}^{n}\right)} \\
& \leq A_{L}\|w\|_{\mathbf{C}^{\mathbf{0}}\left(J ; \mathbf{L}^{1}\left(\mathbb{R}^{+} ; \mathbb{R}^{n}\right)\right)}+C_{\infty} \\
& \leq A_{L} K_{1}+C_{\infty}
\end{aligned}
$$

so that $m_{i}$ is a Caratheodory function. Finally,

$$
\begin{aligned}
\sup _{t \in J} \operatorname{TV}\left(m_{i}(t, \cdot) ; \mathbb{R}^{+}\right) & \leq \sup _{t \in J} \operatorname{TV}\left(\alpha_{i}[w(t)] ; \mathbb{R}^{+}\right)+\sup _{t \in J} \operatorname{TV}\left(\gamma_{i}(t, \cdot) ; \mathbb{R}^{+}\right) \\
& \leq A_{L}\|w\|_{\mathbf{C}^{\mathbf{0}}\left(J ; \mathbf{L}^{\mathbf{1}}\left(\mathbb{R}^{+} ; \mathbb{R}\right)\right)}+C_{\infty} \\
& \leq A_{L} K_{1}+C_{\infty}
\end{aligned}
$$

completing the proof of (m) with

$$
M=A_{L} K_{1}+C_{\infty}
$$

(f) holds. Compute the terms in the right hand side of

$$
\begin{aligned}
\left\|f_{i}\left(t_{2}\right)-f_{i}\left(t_{1}\right)\right\|_{\mathbf{L}^{\mathbf{1}}\left(\mathbb{R}^{+} ; \mathbb{R}\right)} \leq & \sum_{j \neq i}\left\|\left(\alpha_{i}\left[w\left(t_{2}\right)\right]\right)_{j} w_{j}\left(t_{2}\right)-\left(\alpha_{i}\left[w\left(t_{1}\right)\right]\right)_{j} w_{j}\left(t_{1}\right)\right\|_{\mathbf{L}^{\mathbf{1}}\left(\mathbb{R}^{+} ; \mathbb{R}\right)} \\
& +\sum_{j \neq i}\left\|\left(\gamma_{i}\left(t_{2}\right)\right)_{j} w_{j}\left(t_{2}\right)-\left(\gamma_{i}\left(t_{1}\right)\right)_{j} w_{j}\left(t_{1}\right)\right\|_{\mathbf{L}^{\mathbf{1}}\left(\mathbb{R}^{+} ; \mathbb{R}\right)}
\end{aligned}
$$


separately, obtaining

$$
\begin{aligned}
& \left\|\left(\alpha_{i}\left[w\left(t_{2}\right)\right]\right)_{j} w_{j}\left(t_{2}\right)-\left(\alpha_{i}\left[w\left(t_{1}\right)\right]\right)_{j} w_{j}\left(t_{1}\right)\right\|_{\mathbf{L}^{\mathbf{1}}\left(\mathbb{R}^{+} ; \mathbb{R}\right)} \\
& \leq\left\|\alpha_{i}\left[w\left(t_{2}\right)\right]\right\|_{\mathbf{C}^{\mathbf{0}}\left(\mathbb{R}^{+} ; \mathbb{R}^{n}\right)}\left\|w\left(t_{2}\right)-w\left(t_{1}\right)\right\|_{\mathbf{L}^{1}\left(\mathbb{R}^{+} ; \mathbb{R}^{n}\right)} \\
& +\left\|\alpha_{i}\left[w\left(t_{2}\right)-w\left(t_{1}\right)\right]\right\|_{\mathbf{C}^{\mathbf{0}\left(\mathbb{R}^{+} ; \mathbb{R}^{n}\right)}}\left\|w\left(t_{1}\right)\right\|_{\mathbf{L}^{1}\left(\mathbb{R}^{+} ; \mathbb{R}^{n}\right)} \\
& \leq A_{L}\left\|w\left(t_{2}\right)\right\|_{\mathbf{L}^{1}\left(\mathbb{R}^{+} ; \mathbb{R}\right)}\left\|w\left(t_{2}\right)-w\left(t_{1}\right)\right\|_{\mathbf{L}^{1}\left(\mathbb{R}^{+} ; \mathbb{R}^{n}\right)} \\
& +A_{L}\left\|w\left(t_{2}\right)-w\left(t_{1}\right)\right\|_{\mathbf{L}^{1}\left(\mathbb{R}^{+} ; \mathbb{R}^{n}\right)}\left\|w\left(t_{1}\right)\right\|_{\mathbf{L}^{1}\left(\mathbb{R}^{+} ; \mathbb{R}\right)} \\
& \leq 2 A_{L} K_{1}\left\|w\left(t_{2}\right)-w\left(t_{1}\right)\right\|_{\mathbf{L}^{1}\left(\mathbb{R}^{+} ; \mathbb{R}^{n}\right)} \text {. }
\end{aligned}
$$

and similarly

$$
\begin{aligned}
& \quad\left\|\left(\gamma_{i}\left(t_{2}\right)\right)_{j} w_{j}\left(t_{2}\right)-\left(\gamma_{i}\left(t_{1}\right)\right)_{j} w_{j}\left(t_{1}\right)\right\|_{\mathbf{L}^{1}\left(\mathbb{R}^{+} ; \mathbb{R}\right)} \\
& \leq\left\|\gamma_{i}\left(t_{2}\right)\right\|_{\mathbf{L}^{\infty}\left(\mathbb{R}^{+} ; \mathbb{R}^{n}\right)}\left\|w\left(t_{2}\right)-w\left(t_{1}\right)\right\|_{\mathbf{L}^{1}\left(\mathbb{R}^{+} ; \mathbb{R}^{n}\right)} \\
& \quad+\left\|w\left(t_{1}\right)\right\|_{\mathbf{L}^{\infty}\left(\mathbb{R}^{+} ; \mathbb{R}^{n}\right)}\left\|\gamma_{i}\left(t_{2}\right)-\gamma_{i}\left(t_{1}\right)\right\|_{\mathbf{L}^{1}\left(\mathbb{R}^{+} ; \mathbb{R}^{n}\right)} \\
& \quad \leq C_{\infty}\left\|w\left(t_{2}\right)-w\left(t_{1}\right)\right\|_{\mathbf{L}^{1}\left(\mathbb{R}^{+} ; \mathbb{R}^{n}\right)}+K_{\infty}\left\|\gamma_{i}\left(t_{2}\right)-\gamma_{i}\left(t_{1}\right)\right\|_{\mathbf{L}^{1}\left(\mathbb{R}^{+} ; \mathbb{R}^{n}\right)}
\end{aligned}
$$

[Use the linearity of $\alpha_{i}$ ]

which show that $f_{i} \in \mathbf{C}^{\mathbf{0}}\left(J ; \mathbf{L}^{\mathbf{1}}\left(\mathbb{R}^{+} ; \mathbb{R}\right)\right)$, by (IBVP.3) and (4.30).

We prove now the $\mathbf{L}^{\mathbf{1}}$ and $\mathbf{L}^{\infty}$ bounds on $f$ :

$$
\begin{aligned}
& \left\|f_{i}\right\|_{\mathbf{L}^{\infty}\left(J ; \mathbf{L}^{\mathbf{1}}\left(\mathbb{R}^{+} ; \mathbb{R}\right)\right)} \\
\leq & \sum_{j \neq i}\left(\left\|\left(\alpha_{i}[w(t)]\right)_{j} w_{j}(t)\right\|_{\mathbf{L}^{\mathbf{1}\left(\mathbb{R}^{+} ; \mathbb{R}\right)}}+\left\|\left(\gamma_{i}(t)\right)_{j} w_{j}\left(t_{2}\right)\right\|_{\mathbf{L}^{\mathbf{1}\left(\mathbb{R}^{+} ; \mathbb{R}\right)}}\right) \\
\leq & \left(A_{L} K_{1}^{2}+C_{\infty} K_{1}\right) n
\end{aligned}
$$

proving the $\mathbf{L}^{\infty}$ bound on $f_{i}$ with

$$
F_{1}=\left(A_{L} K_{1}^{2}+C_{\infty} K_{1}\right) n .
$$

The $\mathbf{L}^{\infty}$ bound is proved similarly:

$$
\begin{aligned}
& \left\|f_{i}\right\|_{\left.\mathbf{L}^{\infty}\left(J \times \mathbb{R}^{+} ; \mathbb{R}\right)\right)} \\
\leq & \sum_{j \neq i}\left(\left\|\left(\alpha_{i}[w]\right)_{j} w_{j}\right\|_{\mathbf{L}^{\infty}\left(J \times \mathbb{R}^{+} ; \mathbb{R}\right)}+\left\|\left(\gamma_{i}\right)_{j} w_{j}\right\|_{\mathbf{L}^{\infty}\left(J \times \mathbb{R}^{+} ; \mathbb{R}\right)}\right) \\
\leq & \left(A_{L} K_{1} K_{\infty}+C_{\infty} K_{\infty}\right) n
\end{aligned}
$$

Moreover,

$$
\begin{aligned}
& \operatorname{TV}\left(f_{i}(t, \cdot) ; \mathbb{R}^{+}\right) \\
\leq & \sum_{j \neq i} \operatorname{TV}\left(\left(\alpha_{i}[w(t)](\cdot)\right)_{j} w_{j}(t, \cdot)\right)+\sum_{j \neq i} \operatorname{TV}\left(\left(\gamma_{i}(t, \cdot)\right)_{j} w_{j}(t, \cdot)\right) \\
\leq & \sum_{j \neq i} \operatorname{TV}\left(\left(\alpha_{i}[w(t)](\cdot)\right)_{j} ; \mathbb{R}^{+}\right)\left\|w_{j}(t)\right\|_{\mathbf{L}^{\infty}\left(\mathbb{R}^{+} ; \mathbb{R}\right)}
\end{aligned}
$$

[By (3.4), (3.8) and (4.30)] 


$$
\begin{aligned}
& \quad+\sum_{j \neq i}\left\|\left(\alpha_{i}[w(t)](\cdot)\right)_{j}\right\|_{\mathbf{L}^{\infty}\left(\mathbb{R}^{+} ; \mathbb{R}\right)} \operatorname{TV}\left(w_{j}(t, \cdot) ; \mathbb{R}^{+}\right) \\
& +\sum_{j \neq i} \operatorname{TV}\left(\left(\gamma_{i}(t)\right)_{j} ; \mathbb{R}^{+}\right)\left\|w_{j}\right\|_{\mathbf{L}^{\infty}\left(J \times \mathbb{R}^{+} ; \mathbb{R}\right)} \\
& \quad+\sum_{j \neq i}\left\|\left(\gamma_{j}(t)_{i}\right)\right\|_{\mathbf{L}^{\infty}\left(\mathbb{R}^{+} ; \mathbb{R}\right)} \mathrm{TV}\left(w_{j}(t) ; \mathbb{R}^{+}\right) \\
& \leq 2 n A_{L} K_{\infty} K_{1}+2 n C_{\infty} K_{\infty}
\end{aligned}
$$

[Use (3.4) and (4.30)]

completing the proof that (f) holds with

$$
F_{\infty}=2 n K_{\infty}\left(C_{\infty}+A_{L} K_{1}\right)
$$

(b) holds for $i=1$. Note that, in this case, $\beta_{1}(t, u)$ is independent of $u$, so that using (3.12), $\operatorname{TV}\left(b_{1} ; J\right)=\operatorname{TV}\left(\beta_{1}(\cdot) ; J\right) \leq B_{\infty}$.

$\mathcal{T} w$ is Lipschitz continuous in time with respect to the $\mathbf{L}^{\mathbf{1}}$ norm. Simply apply (SP.7), observing that (f), (g), (m) and (b) were proved above exhibiting bounds that hold uniformly on $\mathcal{X}^{n}$, once the norm of the initial datum $u_{o}$ and the constants in (IBVP.1) (IBVP.5) are fixed.

$(\mathcal{T}(w))_{1} \in \mathcal{X} . \quad$ By Lemma 4.5, $u_{1}$ is well defined as solution to the Initial Boundary Value Problem (4.31) with $i=1$. By (SP.2), (IBVP.4) and (3.10), we have that

$$
\begin{aligned}
\left\|u_{1}\right\|_{\mathbf{C}^{0}\left(J ; \mathbf{L}^{1}\left(\mathbb{R}^{+} ; \mathbb{R}\right)\right)} & \leq\left[\left\|u_{1}^{o}\right\|_{\mathbf{L}^{\mathbf{1}}\left(\mathbb{R}^{+} ; \mathbb{R}\right)}+\frac{1}{\check{g}}\left\|\beta_{1}\right\|_{\mathbf{L}^{1}(J ; \mathbb{R})}+\left(A_{1} K_{1}{ }^{2}+C_{\infty} K_{1}\right) n t_{*}\right] e^{\left(A_{L} K_{1}+C_{\infty}\right) t_{*}} \\
& \leq\left[\left\|u_{1}^{o}\right\|_{\mathbf{L}^{\mathbf{1}\left(\mathbb{R}^{+} ; \mathbb{R}\right)}}+\frac{B_{1}}{\check{g}}+\left(A_{1} K_{1}^{2}+C_{\infty} K_{1}\right) n t_{*}\right] e^{\left(A_{L} K_{1}+C_{\infty}\right) t_{*}} \\
& \leq K_{1} \quad \text { provided } t_{*} \text { is small, since by (4.29)} K_{1}>\left\|u_{1}^{o}\right\|_{\mathbf{L}^{1}\left(\mathbb{R}^{+} ; \mathbb{R}\right)}+\frac{B_{1}}{\check{g}} .
\end{aligned}
$$

To bound the $\mathbf{L}^{\infty}$ norm, Use (SP.2) and (3.11) to obtain

$$
\begin{aligned}
\left\|u_{1}(\tau)\right\|_{\mathbf{L}^{\infty}\left(\left[0, t_{*}\right] \times \mathbb{R}^{+} ; \mathbb{R}\right)} & \leq\left(\left\|u_{1}^{o}\right\|_{\mathbf{L}^{\infty}\left(\mathbb{R}^{+} ; \mathbb{R}\right)}+\frac{B_{\infty}}{\check{g}}+2 n K_{\infty}\left(C_{\infty}+A_{L} K_{1}\right) t_{*}\right) e^{\left(G_{1}+A_{L} K_{1}+C_{\infty}\right) t_{*}} \\
& <K_{\infty} \quad \text { provided } t_{*} \text { is small, since } K_{\infty}>\left\|u_{1}^{o}\right\|_{\mathbf{L}^{\infty}\left(\mathbb{R}^{+} ; \mathbb{R}\right)}+\frac{B_{\infty}}{\check{g}} .
\end{aligned}
$$

Using (SP.6), we have $u_{1} \in \mathbf{C}^{\mathbf{0}}\left(J ; \mathbf{L}^{\mathbf{1}}\left(\mathbb{R}^{+} ; \mathbb{R}\right)\right)$. By (SP.3) and (IBVP.4), for $t \in J$, we have

$$
\begin{aligned}
\operatorname{TV}\left(u_{1}(t) ; \mathbb{R}^{+}\right) \leq & \mathcal{H}(t)\left(F_{\infty} t+\frac{\left\|\beta_{1}\right\|_{\mathbf{L}^{\infty}([0, t] ; \mathbb{R})}+\operatorname{TV}\left(\beta_{1} ; \mathbb{R}^{+}\right)}{\check{g}}+\left\|u_{1}^{o}\right\|_{\mathbf{L}^{\infty}\left(\mathbb{R}^{+} ; \mathbb{R}\right)}+\operatorname{TV}\left(u_{1}^{o} ; \mathbb{R}^{+}\right)\right) \\
\leq & \mathcal{H}\left(t_{*}\right)\left(2 n K_{\infty}\left(C_{\infty}+A_{L} K_{1}\right) t_{*}+\frac{2}{\check{g}} B_{\infty}+\left\|u_{1}^{o}\right\|_{\mathbf{L}^{\infty}\left(\mathbb{R}^{+} ; \mathbb{R}\right)}+\operatorname{TV}\left(u_{1}^{o} ; \mathbb{R}^{+}\right)\right) \\
\leq & K_{\infty} \quad \text { provided } t_{*} \text { is small, since by }(4.29) \\
& K_{\infty}>\left(5+\frac{G_{\infty}}{\check{g}}\right)\left(\frac{2 B_{\infty}}{\check{g}}+\left\|u_{o}\right\|_{\mathbf{L}^{\infty}\left(\mathbb{R}^{+} ; \mathbb{R}^{n}\right)}+\mathrm{TV}\left(u_{o} ; \mathbb{R}^{+}\right)\right),
\end{aligned}
$$


completing the proof that $u_{1}=(\mathcal{T} w)_{1} \in \mathcal{X}$. Remark for later use that, by (SP.4),

$$
u_{1}\left(\cdot, \bar{x}_{1}\right) \in \mathbf{B V}(J ; \mathbb{R}) .
$$

(b) holds for $i>1$. Fix now an index $i>1$, assume that $u_{1}, \ldots, u_{i-1} \in \mathcal{X}$ and consider the Initial Boundary Value Problem (4.31) -(4.32). By (IBVP.4), the function $\beta_{i}$ depends only on $t$ and on $u_{1}, \cdots, u_{i-1}$. Moreover, by (IBVP.4) and (4.36), which hold for every $u_{j}$ with $j<i$, the map

$$
t \mapsto b_{i}(t)=\beta_{i}\left(t, u_{1}\left(t, \bar{x}_{1}\right), \cdots, u_{i-1}\left(t, \bar{x}_{i-1}\right)\right)
$$

is of bounded variation and hence satisfies (b).

$(\mathcal{T}(w))_{i} \in \mathcal{X}$ for $i>1$. Lemma 4.5 implies that there exists a solution $u_{i}$ to (4.31). By (SP.2) and (3.9) (3.10), we have that

$$
\begin{aligned}
& \left\|u_{i}(t)\right\|_{\mathbf{C}^{\mathbf{0}}\left(J ; \mathbf{L}^{1}\left(\mathbb{R}^{+} ; \mathbb{R}\right)\right)} \\
\leq & {\left[\left\|u_{i}^{o}\right\|_{\mathbf{L}^{1}\left(\mathbb{R}^{+} ; \mathbb{R}\right)}+\left\|b_{i}\right\|_{\mathbf{L}^{1}(J ; \mathbb{R})}+F_{1} t_{*}\right] e^{M t_{*}} } \\
\leq & {\left[\left\|u_{i}^{o}\right\|_{\mathbf{L}^{1}\left(\mathbb{R}^{+} ; \mathbb{R}\right)}+B_{1}+B_{L} \sum_{j=1}^{i-1} \int_{0}^{t_{*}}\left|u_{j}\left(s, \bar{x}_{j}\right)\right| \mathrm{d} s+n\left(A_{L} K_{1}^{2}+C_{\infty} K_{1}\right) t_{*}\right] e^{\left(A_{L} K_{1}+C_{\infty}\right) t_{*}} } \\
\leq & {\left[\left\|u_{i}^{o}\right\|_{\mathbf{L}^{1}\left(\mathbb{R}^{+} ; \mathbb{R}\right)}+B_{1}+(i-1) B_{L} K_{\infty} t_{*}+n\left(A_{L} K_{1}^{2}+C_{\infty} K_{1}\right) t_{*}\right] e^{\left(A_{L} K_{1}+C_{\infty}\right) t_{*}} } \\
\leq & K_{1} \text { provided } t_{*} \text { is small, since by (44.29) } K_{1}>\left\|u_{i}^{o}\right\|_{\mathbf{L}^{1}\left(\mathbb{R}^{+} ; \mathbb{R}\right)}+B_{1} .
\end{aligned}
$$

Using [SP.6)] $u_{i} \in \mathbf{C}^{\mathbf{0}}\left(J ; \mathbf{L}^{\mathbf{1}}\left(\mathbb{R}^{+} ; \mathbb{R}\right)\right)$. To bound the $\mathbf{L}^{\infty}$ norm, Use (SP.2), (4.9), (3.9)), and (3.11) to obtain

$$
\begin{aligned}
& \left\|u_{i}\right\|_{\mathbf{L}^{\infty}\left(J \times \mathbb{R}^{+} ; \mathbb{R}\right)} \\
\leq & \left(\left\|u_{i}^{o}\right\|_{\mathbf{L}^{\infty}\left(\mathbb{R}^{+} ; \mathbb{R}\right)}+\frac{\left\|b_{i}\right\|_{\mathbf{L}^{\infty}(J ; \mathbb{R})}}{\check{g}}+F_{\infty} t_{*}\right) e^{\left(G_{1}+M\right) t_{*}} \\
\leq & \left(\left\|u_{i}^{o}\right\|_{\mathbf{L}^{\infty}\left(\mathbb{R}^{+} ; \mathbb{R}\right)}+\frac{B_{\infty}}{\check{g}}+\frac{B_{L}}{\check{g}} \sum_{j=1}^{i-1}\left|u_{j}\left(t, \bar{x}_{j}\right)\right|+2 n K_{\infty}\left(C_{\infty}+A_{L} K_{1}\right) t_{*}\right) e^{\left(G_{1}+A_{L} K_{1}+C_{\infty}\right) t_{*}} \\
\leq & \left(\left(1+\frac{n B_{L}}{\check{g}} e^{\left(G_{1}+A_{L} K_{1}+C_{\infty}\right) t_{*}}\right)\left\|u_{o}\right\|_{\mathbf{L}^{\infty}\left(\mathbb{R}^{+} ; \mathbb{R}^{n}\right)}\right. \\
& \left.\quad+\frac{B_{\infty}}{\check{g}}+\frac{2 n B_{L} K_{\infty}}{\check{g}}\left(C_{\infty}+A_{L} K_{1}\right) t_{*} e^{\left(G_{1}+A_{L} K_{1}+C_{\infty}\right) t_{*}}+2 n K_{\infty}\left(C_{\infty}+A_{L} K_{1}\right) t_{*}\right) \\
& \left.\quad \times e^{\left(G_{1}+A_{L} K_{1}+C_{\infty}\right) t_{*}}\right) \\
< & K_{\infty} \quad \operatorname{provided} t_{*} \text { is small, since by (4.29)} K_{\infty}>\left(1+\frac{n B_{L}}{\check{g}}\right)\left\|u_{o}\right\|_{\mathbf{L}^{\infty}\left(\mathbb{R} ; \mathbb{R}^{n}\right)}+\frac{B_{\infty}}{\check{g}} .
\end{aligned}
$$

We pass now to estimate the total variation. By (SP.3) and (IBVP.4) for $t \in J$, we have

$$
\operatorname{TV}\left(u_{i}(t) ; \mathbb{R}^{+}\right)
$$




$$
\begin{aligned}
& \leq \mathcal{H}(t)\left(F_{\infty} t+\frac{\left\|b_{i}\right\|_{\mathbf{L}^{\infty}([0, t] ; \mathbb{R})}+\mathrm{TV}\left(b_{i} ; J\right)}{\check{g}}+\left\|u_{i}^{o}\right\|_{\mathbf{L}^{\infty}\left(\mathbb{R}^{+} ; \mathbb{R}\right)}+\operatorname{TV}\left(u_{i}^{o} ; \mathbb{R}^{+}\right)\right) \\
& \leq \mathcal{H}\left(t_{*}\right)\left(2 n K_{\infty}\left(C_{\infty}+A_{L} K_{1}\right) t_{*}+\frac{1}{\check{g}}\left(2 B_{\infty}+B_{L} \sum_{j=1}^{i-1}\left(\left\|u_{j}\left(\cdot, \bar{x}_{j}\right)\right\|_{\mathbf{L}^{\infty}(J ; \mathbb{R})}+\operatorname{TV}\left(u_{j}\left(\cdot, \bar{x}_{j}\right) ; J\right)\right)\right)\right. \\
& +\left\|u_{i}^{o}\right\|_{\mathbf{L}^{\infty}\left(\mathbb{R}^{+} ; \mathbb{R}\right)}+\operatorname{TV}\left(u_{i}^{o} ; \mathbb{R}^{+}\right) \\
& \leq \mathcal{H}\left(t_{*}\right)\left(2 n K_{\infty}\left(C_{\infty}+A_{L} K_{1}\right) t_{*}+\frac{2 B_{\infty}}{\check{g}}\right. \\
& +\frac{n B_{L}}{\check{g}}\left(\left\|u^{o}\right\|_{\mathbf{L}^{\infty}\left(\mathbb{R}^{+} ; \mathbb{R}^{n}\right)}+2 n K_{\infty}\left(C_{\infty}+A_{L} K_{1}\right) t_{*}\right) e^{\left(G_{1}+A_{L} K_{1}+C_{\infty}\right) t_{*}} \\
& +\frac{n B_{L}}{\check{g}}\left[\mathrm{TV}\left(u_{o} ; \mathbb{R}^{+}\right)+2\left(G_{1}+A_{L} K_{1}+C_{\infty}\right)\left\|u_{o}\right\|_{\mathbf{L}^{\infty}\left(\mathbb{R}^{+} ; \mathbb{R}^{n}\right)} t_{*}\right] e^{\left(G_{1}+A_{L} K_{1}+C_{\infty}\right) t_{*}} \\
& +\frac{8 n^{2} B_{L}}{\check{g}}\left[1+\left(G_{1}+A_{L} K_{1}+C_{\infty}\right) t_{*}\right] K_{\infty}\left(A_{L} K_{1}+C_{\infty}\right) t_{*} e^{\left(G_{1}+A_{L} K_{1}+C_{\infty}\right) t_{*}} \\
& \left.+\left\|u_{o}\right\|_{\mathbf{L}^{\infty}\left(\mathbb{R}^{+} ; \mathbb{R}^{n}\right)}+\operatorname{TV}\left(u_{o} ; \mathbb{R}^{+}\right)\right)
\end{aligned}
$$

$<K_{\infty} \quad$ provided $t_{*}$ is small, since by (4.29)

$$
K_{\infty}>\left(5+\frac{G_{\infty}}{\check{g}}\right)\left(\frac{2 B_{\infty}}{\check{g}}+\frac{n B_{L}}{\check{g}}\left(\left\|u_{o}\right\|_{\mathbf{L}^{\infty}\left(\mathbb{R}^{+} ; \mathbb{R}^{n}\right)}+\mathrm{TV}\left(u_{o} ; \mathbb{R}^{+}\right)\right)\right)
$$

This concludes the proof of the well posedness of $\mathcal{T}$.

The map $\mathcal{T}$ is a contraction. Fix $w, \bar{w} \in \mathcal{X}$. For later use, we prepare some estimates. By (IBVP.2) and (4.30), for every $i \in\{1, \cdots, n\}$, we deduce that

$$
\left\|\left(\alpha_{i}[w]\right)_{i}-\left(\alpha_{i}[\bar{w}]\right)_{i}\right\|_{\mathbf{L}^{\infty}\left(J \times \mathbb{R}^{+} ; \mathbb{R}\right)} \leq A_{L} d_{\mathcal{X}^{n}}(w, \bar{w}) .
$$

Moreover, by (IBVP.2) and (4.30), for every $i \in\{1, \cdots, n\}$ and $j \in\{1, \cdots, n\} \backslash\{i\}$, we obtain

$$
\begin{aligned}
& \left\|\left(\alpha_{i}[w]\right)_{j} w_{j}-\left(\alpha_{i}[\bar{w}]\right)_{j} \bar{w}_{j}\right\|_{\mathbf{L}^{1}\left(J \times \mathbb{R}^{+} ; \mathbb{R}\right)} \\
& \leq\left\|\left(\alpha_{i}[w]\right)_{j} w_{j}-\left(\alpha_{i}[\bar{w}]\right)_{j} w_{j}\right\|_{\mathbf{L}^{1}\left(J \times \mathbb{R}^{+} ; \mathbb{R}\right)}+\left\|\left(\alpha_{i}[\bar{w}]\right)_{j}\left(w_{j}-\bar{w}_{j}\right)\right\|_{\mathbf{L}^{1}\left(J \times \mathbb{R}^{+} ; \mathbb{R}\right)} \\
& \leq\left\|\left(\alpha_{i}[w-\bar{w}]\right)_{j}\right\|_{\mathbf{L}^{\infty}\left(J \times \mathbb{R}^{+} ; \mathbb{R}\right)} d_{\mathcal{X}^{n}}(w, 0) t_{*}+\left\|\left(\alpha_{i}[\bar{w}]\right)_{j}\right\|_{\mathbf{L}^{\infty}\left(J \times \mathbb{R}^{+} ; \mathbb{R}\right)} t_{*} d_{\mathcal{X}^{n}}(w, \bar{w}) \\
& \leq 2 A_{L} K_{1} t_{*} d_{\mathcal{X}^{n}}(w, \bar{w}) \text {. }
\end{aligned}
$$

Finally by (IBVP.3), for every $i \in\{1, \cdots, n\}$ and $j \in\{1, \cdots, n\} \backslash\{i\}$, we also have

$$
\left\|\left(\gamma_{i}\right)_{j} w_{j}-\left(\gamma_{i}\right)_{j} \bar{w}_{j}\right\|_{\mathbf{L}^{1}\left(J \times \mathbb{R}^{+} ; \mathbb{R}\right)} \leq C_{\infty} t_{*} d_{\mathcal{X}^{n}}(w, \bar{w}) .
$$

For $i=1$ and $t \in J$, Lemma 4.7 implies that

$$
\left\|(\mathcal{T} w)_{1}(t, \cdot)-(\mathcal{T} \bar{w})_{1}(t, \cdot)\right\|_{\mathbf{L}^{1}\left(\mathbb{R}^{+} ; \mathbb{R}\right)}
$$




$$
\begin{aligned}
& \leq 2 e^{2\left(G_{1}+A_{L} K_{1}+C_{\infty}\right) t_{*}} \sum_{j=2}^{n}\left\|\left(\alpha_{1}[w]\right)_{j} w_{j}-\left(\alpha_{1}[\bar{w}]\right)_{j} \bar{w}_{j}\right\|_{\mathbf{L}^{1}\left(J \times \mathbb{R}^{+} ; \mathbb{R}\right)} \\
& +2 e^{2\left(G_{1}+A_{L} K_{1}+C_{\infty}\right) t_{*}} \sum_{j=2}^{n}\left\|\left(\gamma_{1}\right)_{j} w_{j}-\left(\gamma_{1}\right)_{j} \bar{w}_{j}\right\|_{\mathbf{L}^{\mathbf{1}}\left(J \times \mathbb{R}^{+} ; \mathbb{R}\right)} \\
& +e^{\left(2 G_{1}+A_{L} K_{1}+C_{\infty}\right) t_{*}}\left[\left\|u_{1}^{o}\right\|_{\mathbf{L}^{1}\left(\mathbb{R}^{+} ; \mathbb{R}\right)}+2 n K_{1}\left(A_{L} K_{1}+C_{\infty}\right) t_{*}+B_{1}\right] \\
& \quad \times t_{*}\left\|\left(\alpha_{1}[w]\right)_{1}-\left(\alpha_{1}[\bar{w}]\right)_{1}\right\|_{\mathbf{L}^{\infty}\left(J \times \mathbb{R}^{+} ; \mathbb{R}\right)^{\prime}}
\end{aligned}
$$

Therefore, using (4.37), (4.38), and (4.39), we get that

$$
\begin{aligned}
& \left\|(\mathcal{T} w)_{1}(t, \cdot)-(\mathcal{T} \bar{w})_{1}(t, \cdot)\right\|_{\mathbf{L}^{1}\left(\mathbb{R}^{+} ; \mathbb{R}\right)} \\
\leq & 4 n e^{2\left(G_{1}+A_{L} K_{1}+C_{\infty}\right) t_{*}} A_{L} K_{1} t_{*} d_{\mathcal{X}^{n}}(w, \bar{w})+2 n e^{2\left(G_{1}+A_{L} K_{1}+C_{\infty}\right) t_{*}} C_{\infty} t_{*} d_{\mathcal{X}^{n}}(w, \bar{w}) \\
& +e^{\left(2 G_{1}+A_{L} K_{1}+C_{\infty}\right) t_{*}}\left[\left\|u_{1}^{o}\right\|_{\mathbf{L}^{1}\left(\mathbb{R}^{+} ; \mathbb{R}\right)}+2 n K_{1}\left(A_{L} K_{1}+C_{\infty}\right) t_{*}+B_{1}\right] A_{L} d_{\mathcal{X}^{n}}(w, \bar{w})
\end{aligned}
$$

and so, choosing $t_{*}$ sufficiently small, we obtain that

$$
\left\|(\mathcal{T} w)_{1}-(\mathcal{T} \bar{w})_{1}\right\|_{\mathbf{C}^{\mathbf{0}}\left(J ; \mathbf{L}^{\mathbf{1}}\left(\mathbb{R}^{+} ; \mathbb{R}\right)\right)} \leq \frac{1}{2 n} d_{\mathcal{X}^{n}}(w, \bar{w}) .
$$

For $i>1$ and $t \in J$, Lemma 4.7 implies that

$$
\begin{aligned}
& \left\|(\mathcal{T} w)_{i}(t, \cdot)-(\mathcal{T} \bar{w})_{i}(t, \cdot)\right\|_{\mathbf{L}^{1}\left(\mathbb{R}^{+} ; \mathbb{R}\right)} \\
& \leq 2 e^{2\left(G_{1}+A_{L} K_{1}+C_{\infty}\right) t_{*}} \sum_{j=1, j \neq i}^{n}\left\|\left(\alpha_{i}[w]\right)_{j} w_{j}-\left(\alpha_{i}[\bar{w}]\right)_{j} \bar{w}_{j}\right\|_{\mathbf{L}^{1}\left(J \times \mathbb{R}^{+} ; \mathbb{R}\right)} \\
& +2 e^{2\left(G_{1}+A_{L} K_{1}+C_{\infty}\right) t_{*}} \sum_{j=2, j \neq i}^{n}\left\|\left(\gamma_{i}\right)_{j} w_{j}-\left(\gamma_{i}\right)_{j} \bar{w}_{j}\right\|_{\mathbf{L}^{\mathbf{1}}\left(J \times \mathbb{R}^{+} ; \mathbb{R}\right)} \\
& +e^{2\left(G_{1}+A_{L} K_{1}+C_{\infty}\right) t_{*}}\left\|b_{i}-\bar{b}_{i}\right\|_{\mathbf{L}^{1}(J ; \mathbb{R})} \\
& +e^{\left(2 G_{1}+A_{L} K_{1}+C_{\infty}\right) t_{*}}\left[\left\|u_{1}^{o}\right\|_{\mathbf{L}^{1}\left(\mathbb{R}^{+} ; \mathbb{R}\right)}+2 n K_{1}\left(A_{L} K_{1}+C_{\infty}\right) t_{*}+\left\|\bar{b}_{i}\right\|_{\mathbf{L}^{1}(J ; \mathbb{R})}\right] \\
& \quad \times t_{*}\left\|\left(\alpha_{i}[w]\right)_{i}-\left(\alpha_{i}[\bar{w}]\right)_{i}\right\|_{\mathbf{L}^{\infty}(J \times \mathbb{R}+\mathbb{R})}
\end{aligned}
$$

where

$$
b_{i}(t)=\beta_{i}\left(t, u_{1}\left(t, \bar{x}_{1}\right), \cdots, u_{i-1}\left(t, \bar{x}_{i-1}\right)\right) \text { and } \bar{b}_{i}(t)=\beta_{i}\left(t, \bar{u}_{1}\left(t, \bar{x}_{1}\right), \cdots, \bar{u}_{i-1}\left(t, \bar{x}_{i-1}\right)\right)
$$

are the boundary terms respectively for $w$ and $\bar{w}$. We thus have that

$$
\begin{aligned}
& \left\|b_{i}-\bar{b}_{i}\right\|_{\mathbf{L}^{1}(J ; \mathbb{R})} \\
\leq & B_{L} \sum_{j=1}^{i-1}\left\|u_{j}\left(\cdot, \bar{x}_{j}\right)-\bar{u}_{j}\left(\cdot, \bar{x}_{j}\right)\right\|_{\mathbf{L}^{1}(J ; \mathbb{R})} \\
\leq & B_{L} \sum_{j=1}^{i-1} e^{\left(G_{1}+A_{L} K_{1}+C_{\infty}\right) t_{*}} t_{*}\left[e^{G_{1} t_{*}}\left\|u_{j}^{o}\right\|_{\mathbf{L}^{1}\left(\mathbb{R}^{+} ; \mathbb{R}\right)}+t_{*}{ }^{2} 2 n K_{\infty}\left(A_{L} K_{1}+C_{\infty}\right)\right]
\end{aligned}
$$




$$
\begin{aligned}
& \times\left\|\left(\alpha_{j}[w]\right)_{j}-\left(\alpha_{j}[\bar{w}]\right)_{j}\right\|_{\mathbf{L}^{\infty}\left(J \times \mathbb{R}^{+} ; \mathbb{R}\right)} \\
+ & e^{\left(2 G_{1}+A_{L} K_{1}+C_{\infty}\right) t_{*}} \sum_{h=1, h \neq j}^{n}\left\|\left(\alpha_{j}[w]\right)_{h} w_{h}-\left(\alpha_{j}[\bar{w}]\right)_{h} \bar{w}_{h}\right\|_{\mathbf{L}^{1}\left(J \times \mathbb{R}^{+} ; \mathbb{R}\right)} \\
+ & e^{\left(2 G_{1}+A_{L} K_{1}+C_{\infty}\right) t_{*}} \sum_{h=1, h \neq j}^{n}\left\|\left(\gamma_{j}\right)_{h} w_{h}-\left(\gamma_{j}\right)_{h} \bar{w}_{h}\right\|_{\mathbf{L}^{\mathbf{1}}\left(J \times \mathbb{R}^{+} ; \mathbb{R}\right)} \\
\leq & B_{L} n e^{\left(G_{1}+A_{L} K_{1}+C_{\infty}\right) t_{*}} t_{*}\left[e^{G_{1} t_{*}}\left\|u_{o}\right\|_{\mathbf{L}^{\mathbf{1}}\left(\mathbb{R}^{+} ; \mathbb{R}^{n}\right)}+t_{*}{ }^{2} 2 n K_{\infty}\left(A_{L} K_{1}+C_{\infty}\right)\right] \\
& \times A_{L} d_{\mathcal{X}^{n}}(w, \bar{w}) \\
+ & e^{\left(2 G_{1}+A_{L} K_{1}+C_{\infty}\right) t_{*}} 2 n A_{L} K_{1} t_{*} d_{\mathcal{X}^{n}}(w, \bar{w}) \\
+ & e^{\left(2 G_{1}+A_{L} K_{1}+C_{\infty}\right) t_{*}} 2 n C_{\infty} t_{*} d_{\mathcal{X}^{n}}(w, \bar{w}) .
\end{aligned}
$$

Moreover

$$
\begin{aligned}
& \left\|\bar{b}_{i}\right\|_{\mathbf{L}^{1}(J ; \mathbb{R})} \\
\leq & B_{L} \sum_{j=1}^{i-1}\left\|u_{j}\left(\cdot, \bar{x}_{j}\right)\right\|_{\mathbf{L}^{1}(J ; \mathbb{R})}+B_{1} \\
\leq & n B_{L} e^{\left(2 G_{1}+A_{L} K_{1}+C_{\infty}\right) t_{*}} t_{*}\left\|u_{o}\right\|_{\mathbf{L}^{\infty}\left(\mathbb{R}^{+} ; \mathbb{R}^{n}\right)} \\
& +2 n^{2} B_{L} e^{\left(G_{1}+A_{L} K_{1}+C_{\infty}\right) t_{*}} K_{\infty}\left(A_{L} K_{1}+C_{\infty}\right) t_{*}{ }^{2} .
\end{aligned}
$$

Finally, using again (4.37), (4.38), and (4.39), we obtain

$$
\begin{aligned}
& \left\|(\mathcal{T} w)_{i}(t, \cdot)-(\mathcal{T} \bar{w})_{i}(t, \cdot)\right\|_{\mathbf{L}^{\mathbf{1}\left(\mathbb{R}^{+} ; \mathbb{R}\right)}} \\
\leq & 4 n A_{L} K_{1} t_{*} d_{\mathcal{X}^{n}}(w, \bar{w}) e^{2\left(G_{1}+A_{L} K_{1}+C_{\infty}\right) t_{*}} \\
& +2 n C_{\infty} t_{*} d_{\mathcal{X}^{n}}(w, \bar{w}) e^{2\left(G_{1}+A_{L} K_{1}+C_{\infty}\right) t_{*}} \\
& +n A_{L} B_{L}\left[e^{G_{1} t_{*}}\left\|u_{o}\right\|_{\mathbf{L}^{\mathbf{1}}\left(\mathbb{R}^{+} ; \mathbb{R}^{n}\right)}+t_{*}{ }^{2} 2 n K_{\infty}\left(A_{L} K_{1}+C_{\infty}\right)\right] t_{*} d_{\mathcal{X}^{n}}(w, \bar{w}) e^{3\left(G_{1}+A_{L} K_{1}+C_{\infty}\right) t_{*}} \\
& +2 n A_{L} K_{1} t_{*} d_{\mathcal{X}^{n}}(w, \bar{w}) e^{4\left(G_{1}+A_{L} K_{1}+C_{\infty}\right) t_{*}} \\
& +2 n C_{\infty} t_{*} d_{\mathcal{X}^{n}}(w, \bar{w}) e^{4\left(G_{1}+A_{L} K_{1}+C_{\infty}\right) t_{*}} \\
& +A_{L}\left[\left\|u_{1}^{o}\right\|_{\mathbf{L}^{\mathbf{1}}\left(\mathbb{R}^{+} ; \mathbb{R}\right)}+2 n K_{1}\left(A_{L} K_{1}+C_{\infty}\right) t_{*}+\left\|\bar{b}_{i}\right\|_{\mathbf{L}^{1}(J ; \mathbb{R})}\right] t_{*} d_{\mathcal{X}^{n}}(w, \bar{w}) e^{\left(2 G_{1}+A_{L} K_{1}+C_{\infty}\right) t_{*}} .
\end{aligned}
$$

Choosing $t_{*}$ sufficiently small, we obtain $\left\|(\mathcal{T} w)_{i}-(\mathcal{T} \bar{w})_{i}\right\|_{\mathbf{C}^{0}\left(J ; \mathbf{L}^{1}\left(\mathbb{R}^{+} ; \mathbb{R}\right)\right)} \leq \frac{1}{2 n} d_{\mathcal{X}^{n}}(w, \bar{w})$. Together with (4.40), this implies that $d_{\mathcal{X}^{n}}(\mathcal{T} w, \mathcal{T} \bar{w}) \leq \frac{1}{2} d_{\mathcal{X}^{n}}(w, \bar{w})$, hence $\mathcal{T}$ is a contraction.

Existence, Uniqueness and Lipschitz Continuity in Time. On the basis of Definition 4.3, a map $u$ is a solution to (3.1) on $\left[0, t_{*}\right]$ if and only if it is a fixed point of $\mathcal{T}$, whence we have existence and uniqueness of the solution on the time interval $\left[0, t_{*}\right]$. The Lipschitz continuity of $u_{*}$ in time follows from (SP.6).

Dependence on the Boundary and Initial Data. Call $u^{\prime}$, respectively $u^{\prime \prime}$, the solution corresponding to the boundary datum $\beta^{\prime}$, respectively $\beta^{\prime \prime}$, and to the initial datum $u_{o}^{\prime}$, respectively $u_{o}^{\prime \prime}$. In the following, the constants $K_{1}$ and $K_{\infty}$ satisfy (4.29) for both $u_{o}^{\prime}$ and $u_{o}^{\prime \prime}$. Fix 
$i \in\{1, \cdots, n\}$ and $t \in\left[0, t_{*}\right]$. Estimate (4.25) implies that

$$
\begin{aligned}
& \left\|u_{i}^{\prime}(t)-u_{i}^{\prime \prime}(t)\right\|_{\mathbf{L}^{\mathbf{1}}\left(\mathbb{R}^{+} ; \mathbb{R}\right)} \leq e^{M t}\left\|u_{o, i}^{\prime}(t)-u_{o, i}^{\prime \prime}(t)\right\|_{\mathbf{L}^{\mathbf{1}}\left(\mathbb{R}^{+} ; \mathbb{R}\right)} \\
& +2 e^{2\left(G_{1}+M\right) t} \int_{0}^{t} \int_{\mathbb{R}^{+}} \sum_{j=1, j \neq i}^{n}\left|\left(\alpha_{i}\left[u^{\prime}(s)\right](x)\right)_{j} u_{j}^{\prime}(s, x)-\left(\alpha_{i}\left[u^{\prime \prime}(s)\right](x)\right)_{j} u_{j}^{\prime \prime}(s, x)\right| \mathrm{d} s \mathrm{~d} x \\
& +2 e^{2\left(G_{1}+M\right) t} \int_{0}^{t} \int_{\mathbb{R}^{+}} \sum_{j=1, j \neq i}^{n}\left|\left(\gamma_{i}(s, x)\right)_{j} \| u_{j}^{\prime}(s, x)-u_{j}^{\prime \prime}(s, x)\right| \mathrm{d} s \mathrm{~d} x \\
& +e^{2\left(G_{1}+M\right) t} \int_{0}^{t}\left|\beta_{i}^{\prime}\left(s, \cdots, u_{i-1}^{\prime}\left(s, \bar{x}_{i-1}-\right)\right)-\beta_{i}^{\prime \prime}\left(s, \cdots, u_{i-1}^{\prime \prime}\left(s, \bar{x}_{i-1}-\right)\right)\right| \mathrm{d} s \\
& +e^{2\left(G_{1}+M\right) t}\left[\left\|u_{o, i}^{\prime \prime}\right\|_{\mathbf{L}^{\infty}\left(\mathbb{R}^{+} ; \mathbb{R}\right)}+2 t F_{\infty}+\frac{1}{\check{g}}\left\|\beta_{i}^{\prime \prime}\left(\cdot, \cdots, u_{i-1}^{\prime \prime}\left(\cdot, \bar{x}_{i-1}-\right)\right)\right\|_{\left.\mathbf{L}^{\infty}([0, t] ; \mathbb{R})\right] \times}\right] \\
& \quad \times \int_{0}^{t}\left\|\left(\alpha_{i}\left[u^{\prime}(s)\right]\right)_{i}-\left(\alpha_{i}\left[u^{\prime \prime}(s)\right]\right)_{i}\right\|_{\mathbf{L}^{\mathbf{1}}\left(\mathbb{R}^{+} ; \mathbb{R}\right)} \mathrm{d} s .
\end{aligned}
$$

We need to estimate every term in the right hand side of (4.42). Preliminary, using (IBVP.4). (3.9) and (4.28), we deduce, for $t \in\left[0, t_{*}\right]$, that

$$
\begin{aligned}
& \int_{0}^{t}\left|\beta_{i}^{\prime}\left(s, \cdots, u_{i-1}^{\prime}\left(s, \bar{x}_{i-1}-\right)\right)-\beta_{i}^{\prime \prime}\left(s, \cdots, u_{i-1}^{\prime \prime}\left(s, \bar{x}_{i-1}-\right)\right)\right| \mathrm{d} s \\
\leq & B_{L} \sum_{j=1}^{i-1}\left\|u_{j}^{\prime}\left(\cdot, \bar{x}_{j}-\right)-u_{j}^{\prime \prime}\left(\cdot, \bar{x}_{j}-\right)\right\|_{\mathbf{L}^{1}((0, t) ; \mathbb{R})}+\left\|\beta_{i}^{\prime}-\beta_{i}^{\prime \prime}\right\|_{\mathbf{L}^{\infty}\left([0, t] \times\left[0, K_{\infty}\right]^{i-1} ; \mathbb{R}\right)} t \\
\leq & B_{L} \frac{e^{\left(G_{1}+M\right) t}}{G_{\infty}} \sum_{j=1}^{i-1}\left[e^{G_{1} t}\left\|u_{o, j}^{\prime}\right\|_{\mathbf{L}^{\infty}\left(\mathbb{R}^{+} ; \mathbb{R}\right)}+t F_{\infty}\right] \times \\
& \times \int_{0}^{t} \int_{0}^{+\infty}\left|\left(\alpha_{j}\left[u^{\prime}(s)\right](x)\right)_{j}-\left(\alpha_{j}\left[u^{\prime \prime}(s)\right](x)\right)_{j}\right| \mathrm{d} x \mathrm{~d} s \\
+ & B_{L} e^{M t}\left\|u_{o}^{\prime}-u_{o}^{\prime \prime}\right\|_{\mathbf{L}^{1}\left(\mathbb{R}^{+} ; \mathbb{R}^{n}\right)}+B_{L} e^{\left(2 G_{1}+M\right) t} \times \\
& \times \sum_{j=1}^{i} \sum_{h=1, h \neq j}^{n} \int_{0}^{t} \int_{0}^{+\infty}\left|\left(\alpha_{j}\left[u^{\prime}(s)\right](x)\right)_{h} u_{h}^{\prime}(s, x)-\left(\alpha_{j}\left[u^{\prime \prime}(s)\right](x)\right)_{h} u_{h}^{\prime \prime}(s, x)\right| \mathrm{d} x \mathrm{~d} s \\
+ & B_{L} e^{\left(2 G_{1}+M\right) t} \sum_{j=1}^{i} \sum_{h=1, h \neq j}^{n} \int_{0}^{t} \int_{0}^{+\infty}\left|\left(\gamma_{j}(s, x)\right)_{h}\right|\left|u_{h}^{\prime}(s, x)-u_{h}^{\prime \prime}(s, x)\right| \mathrm{d} x \mathrm{~d} s \\
+ & \left\|\beta_{i}^{\prime}-\beta_{i}^{\prime \prime}\right\|_{\mathbf{L}^{\infty}\left([0, t] \times\left[0, K_{\infty}\right]^{i-1} ; \mathbb{R}\right)} t .
\end{aligned}
$$


For $j, h \in\{1, \cdots, n\}, j \neq h$, and $t \in\left[0, t_{*}\right]$, using (IBVP.2), (3.4), (3.5), and (4.29), we have

$$
\begin{aligned}
& \int_{0}^{t} \int_{\mathbb{R}^{+}}\left|\left(\alpha_{j}\left[u^{\prime}(s)\right](x)\right)_{h} u_{h}^{\prime}(s, x)-\left(\alpha_{j}\left[u^{\prime \prime}(s)\right](x)\right)_{h} u_{h}^{\prime \prime}(s, x)\right| \mathrm{d} x \mathrm{~d} s \\
\leq & \int_{0}^{t} \int_{\mathbb{R}^{+}}\left|\left(\alpha_{j}\left[u^{\prime}(s)\right](x)\right)_{h}\left(u_{h}^{\prime}(s, x)-u_{h}^{\prime \prime}(s, x)\right)\right| \mathrm{d} x \mathrm{~d} s \\
& +\int_{0}^{t} \int_{\mathbb{R}^{+}}\left|\left(\alpha_{j}\left[u^{\prime}(s)-u^{\prime \prime}(s)\right](x)\right)_{h} u_{h}^{\prime \prime}(s, x)\right| \mathrm{d} x \mathrm{~d} s \\
\leq & A_{L} K_{1} \int_{0}^{t}\left\|u_{h}^{\prime}(s)-u_{h}^{\prime \prime}(s)\right\|_{\mathbf{L}^{1}\left(\mathbb{R}^{+} ; \mathbb{R}\right)} \mathrm{d} s+A_{1} K_{\infty} \int_{0}^{t}\left\|u^{\prime}(s)-u^{\prime \prime}(s)\right\|_{\mathbf{L}^{1}\left(\mathbb{R}^{+} ; \mathbb{R}^{n}\right)} \mathrm{d} s .
\end{aligned}
$$

Moreover, for $j, h \in\{1, \cdots, n\}, j \neq h$, and $t \in\left[0, t_{*}\right]$, using (IBVP.3), we get

$$
\int_{0}^{t} \int_{\mathbb{R}^{+}}\left|\left(\gamma_{j}(s, x)\right)_{h}\right|\left|u_{h}^{\prime}(s, x)-u_{h}^{\prime \prime}(s, x)\right| \mathrm{d} x \mathrm{~d} s \leq C_{\infty} \int_{0}^{t}\left\|u_{h}^{\prime}(s)-u_{h}^{\prime \prime}(s)\right\|_{\mathbf{L}^{1}\left(\mathbb{R}^{+} ; \mathbb{R}\right)} \mathrm{d} s .
$$

Finally, for $j \in\{1, \cdots, n\}$ and $t \in\left[0, t_{*}\right]$, using (IBVP.2) and (3.5), we have that

$$
\begin{aligned}
\int_{0}^{t}\left\|\left(\alpha_{j}\left[u^{\prime}(s)\right]\right)_{j}-\left(\alpha_{j}\left[u^{\prime \prime}(s)\right]\right)_{j}\right\|_{\mathbf{L}^{1}\left(\mathbb{R}^{+} ; \mathbb{R}\right)} \mathrm{d} s & =\int_{0}^{t} \int_{0}^{+\infty}\left|\left(\alpha_{j}\left[u^{\prime}(s)-u^{\prime \prime}(s)\right]\right)_{j}(x)\right| \mathrm{d} x \mathrm{~d} s \\
& \leq A_{1} \int_{0}^{t}\left\|u^{\prime}(s)-u^{\prime \prime}(s)\right\|_{\mathbf{L}^{1}\left(\mathbb{R}^{+} ; \mathbb{R}^{n}\right)} \mathrm{d} s .
\end{aligned}
$$

Inserting (4.43), (4.44), (4.45), and (4.46) into (4.42) we obtain that, for $t \in\left[0, t_{*}\right]$,

$$
\begin{aligned}
\left\|u^{\prime}(t)-u^{\prime \prime}(t)\right\|_{\mathbf{L}^{1}\left(\mathbb{R}^{+} ; \mathbb{R}^{n}\right)} \leq & \mathcal{H}_{1}(t) \int_{0}^{t}\left\|u^{\prime}(s)-u^{\prime \prime}(s)\right\|_{\mathbf{L}^{1}\left(\mathbb{R}^{+} ; \mathbb{R}^{n}\right)} \mathrm{d} s \\
& +\mathcal{H}_{2}(t)\left\|u_{o}^{\prime}(t)-u_{o}^{\prime \prime}(t)\right\|_{\mathbf{L}^{1}\left(\mathbb{R}^{+} ; \mathbb{R}^{n}\right)} \\
& +e^{2\left(G_{1}+M\right) t}\left\|\beta^{\prime}-\beta^{\prime \prime}\right\|_{\mathbf{L}^{\infty}\left([0, t] \times\left[0, K_{\infty}\right]^{n} ; \mathbb{R}^{n}\right)} t,
\end{aligned}
$$

where

$$
\begin{aligned}
\mathcal{H}_{1}(t)= & n e^{2\left(G_{1}+M\right) t}\left[2 A_{L} K_{1}+2 n A_{1} K_{\infty}+2 C_{\infty}\right] \\
& +n^{2} e^{3\left(G_{1}+M\right) t} \frac{B_{L} A_{1}}{G_{\infty}}\left[e^{G_{1} t}\left\|u_{o}^{\prime}\right\|_{\mathbf{L}^{\infty}\left(\mathbb{R}^{+} ; \mathbb{R}^{n}\right)}+t F_{\infty}\right] \\
& +n^{2} e^{\left(4 G_{1}+3 M\right) t} B_{L}\left[A_{L} K_{1}+n A_{1} K_{\infty}+C_{\infty}\right] \\
& +n^{2} e^{2\left(G_{1}+M\right) t} A_{1}\left[\left\|u_{o}^{\prime \prime}\right\|_{\mathbf{L}^{\infty}\left(\mathbb{R}^{+} ; \mathbb{R}^{n}\right)}+2 n t F_{\infty}+\frac{n}{\check{g}}\left(B_{\infty}+n B_{L} K_{\infty}\right)\right] ; \\
\mathcal{H}_{2}(t)= & n e^{M t}\left(1+B_{L} e^{2\left(G_{1}+M\right) t}\right) .
\end{aligned}
$$

An application of Gronwall Lemma yields (3.14). 
Proof of Corollary 3.3. We proceed with the same notation used in the proof of Theorem 3.1, $u$ being the solution to (3.1) on $J$.

The positivity of each $u_{i}$ directly follows from (4.9)-4.10).

Assume, by contradiction, that there exists a maximal time of existence $\bar{t}$ for the solution $u$ to (3.1). A direct consequence of (NEG) and (EQ) is that

$$
\partial_{t}\left(\sum_{i=1}^{n} u_{i}\right)+\partial_{x}\left(g(t, x) \sum_{i=1}^{n} u_{i}\right)=\left(\sum_{i=1}^{n}\left(\alpha_{i}[u(t)]+\gamma_{i}(t, x)\right)\right) \cdot u \leq 0 .
$$

Therefore, (SP.2) and (SP.3) ensure that $\left\|\sum_{i=1}^{n} u_{i}(t)\right\|_{\mathbf{L}^{1}\left(\mathbb{R}^{+} ; \mathbb{R}\right)},\left\|\sum_{i=1}^{n} u_{i}(t)\right\|_{\mathbf{L}^{\infty}\left(\mathbb{R}^{+} ; \mathbb{R}\right)}$ and $\mathrm{TV}\left(\sum_{i=1}^{n} u_{i}(t) ; \mathbb{R}^{+}\right)$are uniformly bounded on $[0, \bar{t}[$. The uniform continuity of $u$ in time ensures that $u$ can be defined also at time $\bar{t}$. A further application of Theorem 3.1 allows to uniquely extend $u$ beyond time $\bar{t}$, yielding the contradiction.

The functional Lipschitz continuous dependence of $u_{*}$ on the initial datum $u_{o}$ and on the boundary inflow $\beta$ now follows from (SP.8) and (SP.10), possibly iterating the estimate (4.27) to comply with the condition $\gamma(t)<\bar{x}$ therein.

\subsection{Proofs Related to Section 2 - About the Models (1.1)-(1.2) and (1.1)-(1.5)}

Proof of Theorem 2.1. The present proof consists in showing that with the present assumptions, Theorem 3.1 and Corollary 3.3 can be applied to (1.1)-(1.2)-(2.1). To this aim, set for notational simplicity $\bar{a}_{0}=0, \bar{a}_{N+1}=+\infty$ and define

$$
\begin{aligned}
& u_{1+3 j}(t, a)=S\left(t, a+\bar{a}_{j}\right) \\
& u_{2+3 j}(t, a)=I\left(t, a+\bar{a}_{j}\right) \\
& u_{3+3 j}(t, a)=R\left(t, a+\bar{a}_{j}\right) \\
& \text { for } \begin{aligned}
& j=0,1, \ldots, N \\
& t \in \mathbb{I} \\
a & \in \mathbb{R}^{+} .
\end{aligned}
\end{aligned}
$$

Set $g_{i}(t, a)=1$ for $i=1, \ldots, n$ and $n=3 N+3$. Define for $j=0,1, \ldots, N$ and $i=1, \ldots, n$

$$
\begin{aligned}
& \begin{aligned}
{\left[\alpha_{1+3 j}[u](a)\right]_{i} } & =\left\{\begin{array}{lr}
-\sum_{\ell=1}^{N+1} \int_{\bar{a}_{\ell-1}}^{\bar{a}_{\ell}} \lambda\left(a+\bar{a}_{j}, a^{\prime}\right) u_{2+3 \ell}\left(a^{\prime}-\bar{a}_{\ell-1}\right) \mathrm{d} a^{\prime} & i=1+3 j \\
0 & \text { otherwise } \\
{\left[\alpha_{2+3 j}[u](a)\right]_{i}} & = \begin{cases}\sum_{\ell=1}^{N+1} \int_{\bar{a}_{\ell-1}}^{\bar{a}_{\ell}} \lambda\left(a+\bar{a}_{j}, a^{\prime}\right) u_{2+3 \ell}\left(a^{\prime}-\bar{a}_{\ell-1}\right) \mathrm{d} a^{\prime} & i=1+3 j \\
0 & \text { otherwise }\end{cases} \\
{\left[\alpha_{3+3 j}[u](a)\right]_{i}} & =0
\end{array}\right.
\end{aligned} \\
& {\left[\gamma_{1+3 j}(t, a)\right]_{i}=\left\{\begin{array}{lr}
-d_{S}\left(t, a+\bar{a}_{j}\right) & i=1+3 j \\
0 & \text { otherwise }
\end{array}\right.} \\
& {\left[\gamma_{2+3 j}(t, a)\right]_{i}=\left\{\begin{array}{lr}
-d_{I}\left(t, a+\bar{a}_{j}\right)-r_{I}\left(t, a+\bar{a}_{j}\right) & i=2+3 j \\
0 & \text { otherwise }
\end{array}\right.} \\
& {\left[\gamma_{3+3 j}(t, a)\right]_{i}= \begin{cases}-d_{R}\left(t, a+\bar{a}_{j}\right) & i=3+3 j \\
r_{I}\left(t, a+\bar{a}_{j}\right) & i=2+3 j \\
0 & \text { otherwise }\end{cases} }
\end{aligned}
$$


Concerning the boundary conditions and transmission relations, we set $\bar{x}_{i}=\bar{a}_{j}-\bar{a}_{j-1}$ for all $i \in\{1, \cdots, n\}$ and for all $j=\{1, \cdots, N+1\}$ such that $i-3(j-1) \in\{1,2,3\}$. Moreover

$$
\begin{aligned}
& \beta_{1}(t, u)=S_{b}(t) \quad \beta_{2}(t, u)=I_{b}(t) \quad \beta_{3}(t, u)=R_{b}(t) \\
& \beta_{3 j+1}(t, u)=\left(1-\eta_{j}(t)\right) u_{3 j-2} \quad \beta_{3 j+2}(t, u)=u_{3 j-1} \quad \beta_{3 j+3}(t, u)=\eta_{j}(t) u_{3 j-2}+u_{3 j}
\end{aligned}
$$

We now verify that the assumptions required in Theorem 3.1 on the functions above hold.

(IBVP.1) holds. It is immediate, since $g_{i}(t, a)=1$ for all $(t, a) \in \mathbb{I} \times \mathbb{R}^{+}$.

(IBVP.2) holds. On the basis of (4.48), we have:

$$
\begin{aligned}
& \left\|\alpha_{i}[w]\right\|_{\mathbf{L}^{\infty}\left(\mathbb{R}^{+} ; \mathbb{R}^{n}\right)} \leq \Lambda_{\infty}\|u\|_{\mathbf{L}^{1}\left(\mathbb{R}^{+} ; \mathbb{R}^{n}\right)} \quad \text { [By (2.2), (3.4) holds with } A_{L}=\Lambda_{\infty} \text { ] } \\
& \operatorname{TV}\left(\alpha_{i}[w] ;\left(\mathbb{R}^{+}\right)^{n}\right) \leq \sum_{\ell=1}^{N+1} \int_{\bar{a}_{\ell-1}}^{\bar{a}_{\ell}} \operatorname{TV}\left(\lambda\left(\cdot, a^{\prime}\right) ; \mathbb{R}^{+}\right)\left|w_{2+3 \ell}\left(a^{\prime}-\bar{a}_{\ell-1}\right)\right| \mathrm{d} a^{\prime} \\
& \leq \Lambda_{\infty}\|u\|_{\mathbf{L}^{1}\left(\mathbb{R}^{+} ; \mathbb{R}^{n}\right)} \\
& \text { [By (2.2), (3.4) holds with } \left.A_{L}=\Lambda_{\infty}\right] \\
& \left\|\alpha_{i}[u]\left(a_{1}\right)-\alpha_{i}[u]\left(a_{2}\right)\right\| \leq \sum_{\ell=1}^{N+1} \int_{\bar{a}_{\ell-1}}^{\bar{a}_{\ell}}\left|\lambda\left(a_{1}+\bar{a}_{j}, a^{\prime}\right)-\lambda\left(a_{2}+\bar{a}_{j}, a^{\prime}\right)\right|\left|u_{2+3 \ell}\right|\left(a^{\prime}-\bar{a}_{\ell-1}\right) \mathrm{d} a^{\prime} \quad[\operatorname{By}[\mathbf{( \lambda )}] \\
& \leq \Lambda_{l} \sum_{\ell=1}^{N+1} \int_{\bar{a}_{\ell-1}}^{\bar{a}_{\ell}}\left|u_{2+3 \ell}\right|\left(a^{\prime}-\bar{a}_{\ell-1}\right) \mathrm{d} a^{\prime}\left|a_{1}-a_{2}\right| \\
& \leq N \Lambda_{L}\|u\|_{\mathbf{L}^{1}\left(\mathbb{R}^{+} ; \mathbb{R}^{n}\right)}\left|a_{1}-a_{2}\right|
\end{aligned}
$$

proving (3.6) in (IBVP.2) with $A_{2}=N \Lambda_{L}\|u\|_{\mathbf{L}^{1}\left(\mathbb{R}^{+} ; \mathbb{R}^{n}\right)}$.

(IBVP.3) holds. Refer to (4.49). The Lipschitz continuity of $\gamma$ directly follows from (2.5), proving (3.7). The other conditions are immediate consequences of (2.4), (2.6) and (2.4).

(IBVP.4) holds. Refer to (4.50). Condition (3.9) is immediate, thanks to the boundedness of $\eta$. (3.10), (3.11) and (3.12) follow from (2.7).

(IBVP.5) holds. It is an immediate consequence of (2.7), using (4.47) at $t=0$.

(POS) holds. It immediately follows from (IB).

(NEG) holds. Long but straightforward computations based on (1.1), (4.48) and (4.49) show that (NEG) holds.

(EQ) holds. It is a direct consequence of (1.1).

Dependence on $\eta$. Theorem 3.1 and Corollary 3.3 ensure the Lipschitz continuous dependence of the solution $(S, I, R)$ in $\mathbf{L}^{\mathbf{1}}$ on the boundary datum through its $\mathbf{L}^{\mathbf{1}}$ norm. In view of (4.50), this yields the continuous dependence of the solution $(S, I, R)$ in $\mathbf{L}^{1}$ on $\eta$ in $\mathbf{L}^{\infty}$. 
The proof of Theorem 2.2 can be obtained from the one above exchanging the roles of the independent variables $t$ and $a$. However, for completeness, we provide an independent proof.

Proof of Theorem 2.2. We now show that Theorem 3.1 can be iteratively applied to problem (1.1)-(2.1)-(1.5). To this aim, define $n=3$ and

$$
\begin{aligned}
& u_{1}(t, a)=S(t, a), \quad u_{2}(t, a)=I(t, a), \quad u_{3}(t, a)=R(t, a) . \\
& {\left[\alpha_{1}[u](a)\right]_{i}=\left\{\begin{array}{ll}
-\int_{\mathbb{R}^{+}} \lambda\left(a, a^{\prime}\right) u_{2}\left(a^{\prime}\right) \mathrm{d} a^{\prime} & i=1 \\
0 & i=2,3
\end{array} \quad\left[\gamma_{1}(t, a)\right]_{i}= \begin{cases}-d_{S}(t, a) & i=1 \\
0 & i=2,3\end{cases} \right.} \\
& {\left[\alpha_{2}[u](a)\right]_{i}=\left\{\begin{array}{ll}
\int_{\mathbb{R}^{+}} \lambda\left(a, a^{\prime}\right) u_{2}\left(a^{\prime}\right) \mathrm{d} a^{\prime} & i=1 \\
0 & i=2,3
\end{array} \quad\left[\gamma_{2}(t, a)\right]_{i}= \begin{cases}-d_{I}(t, a)-r_{I}(t, a) & i=2 \\
0 & i=1,3\end{cases} \right.} \\
& {\left[\alpha_{3}(a)\right]_{i}=0} \\
& {\left[\gamma_{3}(t, a)\right]_{i}= \begin{cases}0 & i=1 \\
r_{I}(t, a) & i=2 \\
-d_{R}(t, a) & i=3\end{cases} }
\end{aligned}
$$

We now iteratively apply Theorem 3.1 on the time interval $\left[\bar{t}_{k}, \bar{t}_{k+1}\right]$ assigning, on the basis of (2.1), the initial and boundary data

$$
\begin{aligned}
& k=0 \quad \begin{cases}u_{1}^{o}(a)=S_{o}(a) & \beta_{1}(t, u)=S_{b}(t)\end{cases} \\
& t \in\left[0, \bar{t}_{1}\right] \quad \begin{cases}u_{2}^{o}(a)=I_{o}(a) & \beta_{2}(t, u)=I_{b}(t) \\
u_{0}(a)\end{cases} \\
& a \in \mathbb{R}^{+} \quad \begin{cases}u_{3}^{o}(a)=R_{o}(a) & \beta_{3}(t, u)=R_{b}(t)\end{cases}
\end{aligned}
$$

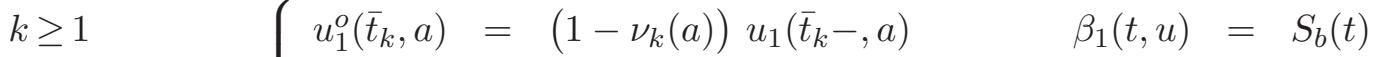

$$
\begin{aligned}
& t \in\left[\bar{t}_{k}, \bar{t}_{k+1}\right] \quad \begin{cases}u_{2}^{o}\left(\bar{t}_{k}, a\right)=u_{2}\left(\bar{t}_{k}-, a\right) & \beta_{2}(t, u)=I_{b}(t)\end{cases} \\
& a \in \mathbb{R}^{+} \quad\left(u_{3}^{o}\left(\bar{t}_{k}, a\right)=u_{3}\left(\bar{t}_{k}-, a\right)+\nu_{k}(a) u_{1}\left(\bar{t}_{k}-, a\right) \quad \beta_{3}(t, u)=R_{b}(t)\right.
\end{aligned}
$$

We now verify that the assumptions required in Theorem 3.1 on the functions above hold.

(IBVP.1) holds. It is immediate, since $g_{i}(t, a)=1$ for all $(t, a) \in \mathbb{I} \times \mathbb{R}^{+}$.

\section{(IBVP.2) holds.}

$$
\begin{array}{rlrl}
\left\|\alpha_{i}[w]\right\|_{\mathbf{L}^{\infty}\left(\mathbb{R}^{+} ; \mathbb{R}^{n}\right)} & \leq \Lambda_{\infty}\|u\|_{\mathbf{L}^{1}\left(\mathbb{R}^{+} ; \mathbb{R}^{n}\right)} & \text { [By (2.2), (3.4) holds with } \left.A_{L}=\Lambda_{\infty}\right] \\
\operatorname{TV}\left(\alpha_{i}[w] ;\left(\mathbb{R}^{+}\right)^{n}\right) & \leq \int_{\mathbb{R}^{+}} \operatorname{TV}\left(\lambda\left(\cdot, a^{\prime}\right) ; \mathbb{R}^{+}\right)\left|w_{j}\left(a^{\prime}\right)\right| \mathrm{d} a^{\prime} & \\
& \leq \Lambda_{\infty}\|u\|_{\mathbf{L}^{1}\left(\mathbb{R}^{+} ; \mathbb{R}^{n}\right)} & {[\text { By 4.5 }]} \\
\left\|\alpha_{i}[u]\left(a_{1}\right)-\alpha_{i}[u]\left(a_{2}\right)\right\| & \leq \int_{\mathbb{R}^{+}}\left|\lambda\left(a_{1}, a^{\prime}\right)-\lambda\left(a_{2}, a^{\prime}\right)\right|\left|u_{2}\right|\left(a^{\prime}\right) \mathrm{d} a^{\prime} \\
& \leq \Lambda_{l} \int_{\mathbb{R}^{+}}\left|u_{2}\left(a^{\prime}\right)\right| \mathrm{d} a^{\prime}\left|a_{1}-a_{2}\right| & \\
& \leq \Lambda_{L}\|u\|_{\mathbf{L}^{1}\left(\mathbb{R}^{+} ; \mathbb{R}^{n}\right)}\left|a_{1}-a_{2}\right| . & {\left[\text { holds with } A_{L}=\Lambda_{\infty}\right]}
\end{array}
$$

(IBVP.3) holds. The Lipschitz continuity of $\gamma$ directly follows from (2.5), proving (3.7). The other conditions are immediate consequences of (2.4), (2.6) and (2.4).

(IBVP.4) holds. The definitions (4.51) and (2.7) directly imply (3.10), (3.11) and (3.12). 
(IBVP.5) holds. It directly follows from (4.51).

(POS) holds. It immediately follows from (IB)

(NEG) holds. Long but straightforward computations based on (1.1), (4.48) and (4.49) show that (NEG) holds.

(EQ) holds. It is a direct consequence of (1.1).

Dependence on $\nu$. Repeat the same argument used in the final part of the proof of Theorem 2.1, replacing the boundary datum with the initial datum.

Acknowledgments: Part of this work was supported by the PRIN 2015 project Hyperbolic Systems of Conservation Laws and Fluid Dynamics: Analysis and Applications and by the GNAMPA 2018 project Conservation Laws: Hyperbolic Games, Vehicular Traffic and Fluid dynamics. The IBM Power Systems Academic Initiative substantially contributed to the numerical integrations.

\section{References}

[1] L. Ambrosio, N. Fusco, and D. Pallara. Functions of bounded variation and free discontinuity problems. Oxford Mathematical Monographs. The Clarendon Press Oxford University Press, New York, 2000.

[2] C. Bardos, A. Y. le Roux, and J.-C. Nédélec. First order quasilinear equations with boundary conditions. Comm. Partial Differential Equations, 4(9):1017-1034, 1979.

[3] A. Bressan. Hyperbolic systems of conservation laws, volume 20 of Oxford Lecture Series in Mathematics and its Applications. Oxford University Press, Oxford, 2000. The one-dimensional Cauchy problem.

[4] A. Bressan and B. Piccoli. Introduction to the mathematical theory of control, volume 2 of AIMS Series on Applied Mathematics. American Institute of Mathematical Sciences (AIMS), Springfield, MO, 2007.

[5] R. M. Colombo and M. Garavello. Stability and optimization in structured population models on graphs. Math. Biosci. Eng., 12(2):311-335, 2015.

[6] R. M. Colombo and M. Garavello. Optimizing vaccination strategies in a SIR model. Preprint, 2019.

[7] A. El-Alami Laaroussi, M. Rachik, and M. Elhia. An optimal control problem for a spatiotemporal SIR model. Int. J. Dyn. Control, 6(1):384-397, 2018.

[8] K. P. Hadeler and J. Müller. Optimal harvesting and optimal vaccination. Math. Biosci., 206(2):249$272,2007$.

[9] H. Inaba. Age-Structured SIR Epidemic Model, pages 287-331. Springer Singapore, Singapore, 2017.

[10] W. O. Kermack, A. G. McKendrick, and G. T. Walker. A contribution to the mathematical theory of epidemics. Proceedings of the Royal Society of London. Series A, Containing Papers of a Mathematical and Physical Character, 115(772):700-721, 1927.

[11] S. N. Kružhkov. First order quasilinear equations with several independent variables. Mat. Sb. (N.S.), 81 (123):228-255, 1970. 
[12] J. Müller. Optimal vaccination patterns in age-structured populations. SIAM J. Appl. Math., 59(1):222-241, 1999.

[13] J. Müller. Optimal vaccination patterns in age-structured populations: endemic case. In Proceedings of the Conference on Dynamical Systems in Biology and Medicine (Veszprém, 1997), volume 31, pages 149-160, 2000.

[14] J. D. Murray. Mathematical biology. I, volume 17 of Interdisciplinary Applied Mathematics. SpringerVerlag, New York, third edition, 2002. An introduction.

[15] B. Perthame. Transport equations in biology. Frontiers in Mathematics. Birkhäuser Verlag, Basel, 2007.

[16] E. Rossi. Definitions of solutions to the IBVP for multi-dimensional scalar balance laws. J. Hyperbolic Differ. Equ., 15(2):349-374, 2018.

[17] D. Serre. Systems of conservation laws. 2. Cambridge University Press, Cambridge, 2000. Geometric structures, oscillations, and initial-boundary value problems, Translated from the 1996 French original by I. N. Sneddon.

[18] X. Wang, H. Peng, B. Shi, D. Jiang, S. Zhang, and B. Chen. Optimal vaccination strategy of a constrained time-varying SEIR epidemic model. Commun. Nonlinear Sci. Numer. Simul., 67:37-48, 2019.

[19] G. Zaman, Y. H. Kang, G. Cho, and I. H. Jung. Optimal strategy of vaccination \& treatment in an sir epidemic model. Math. Comput. Simulation, 136:63-77, 2017. 